University of Texas at El Paso

\title{
DigitalCommons@UTEP
}

Open Access Theses \& Dissertations

2016-01-01

\section{Treadmill-Based Perturbation Training For Preventing Falls Among Young Adults}

Patrick Anthony Cereceres

University of Texas at El Paso, patrick.cereceres@gmail.com

Follow this and additional works at: https://digitalcommons.utep.edu/open_etd

Part of the Biomechanics Commons, and the Medicine and Health Sciences Commons

\section{Recommended Citation}

Cereceres, Patrick Anthony, "Treadmill-Based Perturbation Training For Preventing Falls Among Young Adults" (2016). Open Access Theses \& Dissertations. 620.

https://digitalcommons.utep.edu/open_etd/620

This is brought to you for free and open access by DigitalCommons@UTEP. It has been accepted for inclusion in Open Access Theses \& Dissertations by an authorized administrator of DigitalCommons@UTEP. For more information, please contact lweber@utep.edu. 


\section{TREADMILL-BASED PERTURBATION TRAINING FOR PREVENTING FALLS}

AMONG YOUNG ADULTS

\section{PATRICK ANTHONY CERECERES}

Master's Program in Kinesiology

APPROVED:

Feng Yang, Ph.D., Chair

João Ferreira-Pinto, Ph.D.

Jason Boyle, Ph.D.

Charles Ambler, Ph.D.

Dean of the Graduate School 
Copyright ()

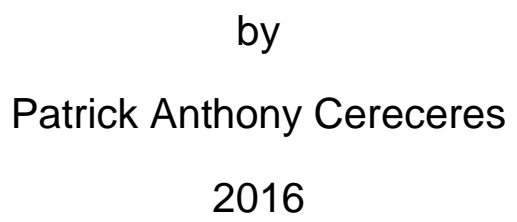




\section{DEDICATION}

This thesis is dedicated to my parents, who have helped me get to where I am today. Without their support, I would not be in this position. Thank you, Mama and Tata. I would also like to dedicate this thesis to my Grandma Mary who has since passed.

While she was not able to see me graduate from college, I know she would be proud that I have made it this far. 
TREADMILL-BASED PERTURBATION TRAINING FOR PREVENTING FALLS AMONG YOUNG ADULTS

by

PATRICK ANTHONY CERECERES, B.S

THESIS

Presented to the Faculty of the Graduate School of

The University of Texas at El Paso

in Partial Fulfillment

of the Requirements

for the Degree of

MASTER OF SCIENCE

Department of Kinesiology

THE UNIVERSITY OF TEXAS AT EL PASO

December 2016 


\section{ACKNOWLEDGEMENTS}

This study was partially funded by a Graduate Student Research Grant from the Programa de Ivestigación en Migracion y Salud (2016 to PAC) and a research grant (2014-070 to FY) from The Retirement Research Foundation. I would also like to acknowledge Dr. Feng Yang for pushing me to work and produce the best study that I could. Without his help, none of this could have been possible. Additionally, I would like to thank Dr. João Ferreira-Pinto and Dr. Jason Boyle for serving on my committee. Their questions and comments were greatly valued. And a special thanks to Christina Carrera, Ivette Sanchez, Fabricio Saucedo, and Zachary Henry for their assistance in collecting data and to Joshua Padilla and Anissa Reynaud for their assistance in processing data. 


\section{ABSTRACT}

Falls present a serious challenge not only among older adults, but for young adults, especially those whose working conditions exposes them to a high fall hazard. Treadmill-based perturbation training, as a task-specific training modality, has emerged as a new paradigm used to prevent falls. However, the training program in previous studies contained up to 30 perturbation trials. It is unclear if a reduced number of perturbation trials could still induce positive effect in preventing falls. The purpose of this study was to investigate the effect of a treadmill-based perturbation training paradigm consisting of 8 slip perturbations on reducing slip-related falls in young adults. Forty-three young adults participated in this study and were randomly assigned to either the training group (those who received treadmill-based perturbation training) or the control group (those who did not receive perturbation but the mocking training on the same treadmill). After the training, both groups were exposed to an unannounced slip during overground walking. The reactions to the overground slip, including the fall incidences and dynamic stability, were compared between groups. The results indicated that the perturbation training reduced the likelihood of falls and improved the dynamic stability at the recovery touchdown down during the overground slip in the training group when compared to the control group. The findings of this study showed that treadmill-based perturbation training, even at a lower dosage, can be effective in preventing falls. However, more research is needed in order to largely deploy this training to both healthy individuals and people with neurological disease. 


\section{TABLE OF CONTENTS}

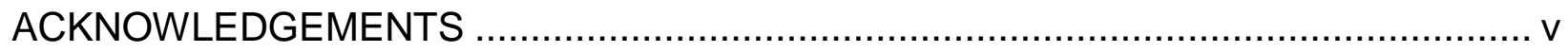

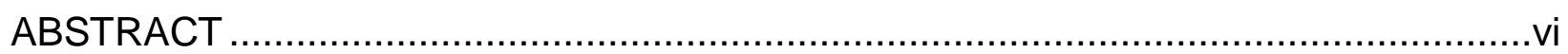

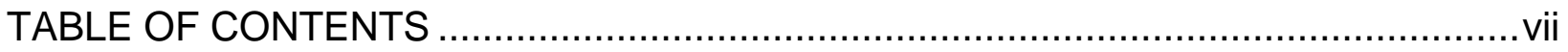

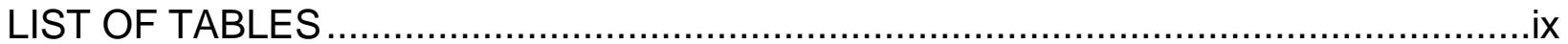

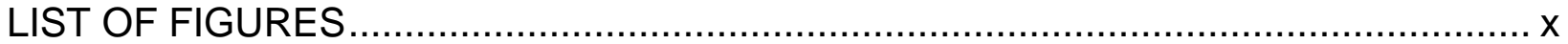

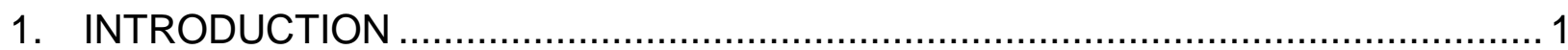

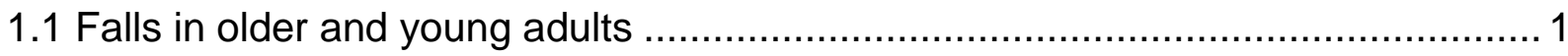

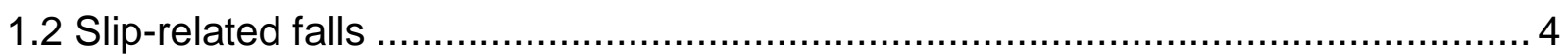

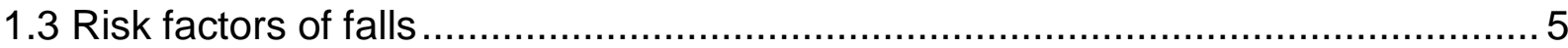

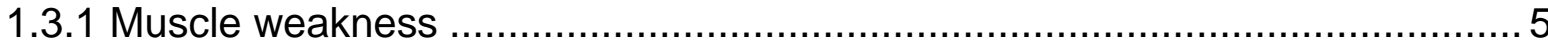

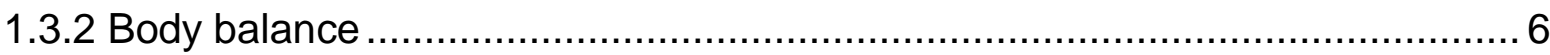

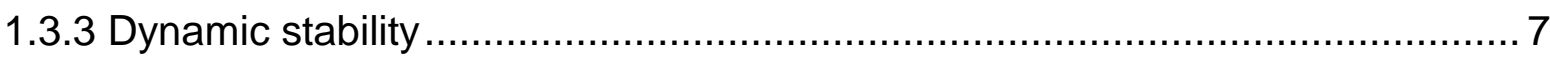

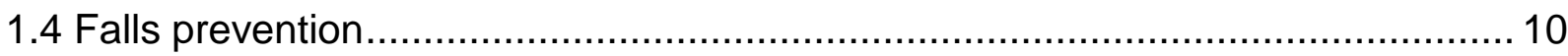

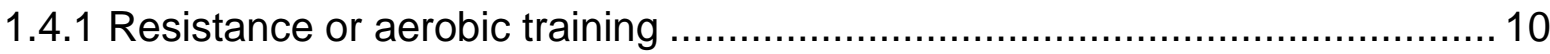

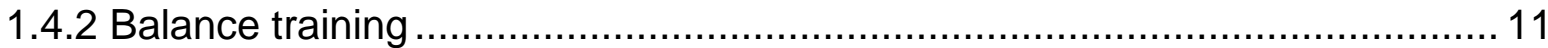

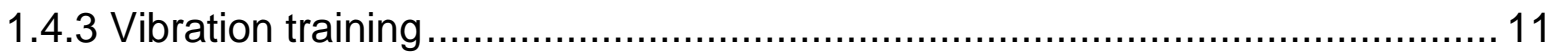

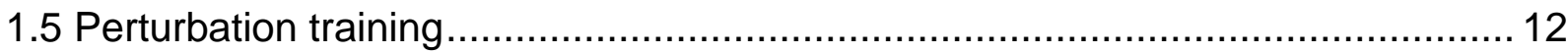

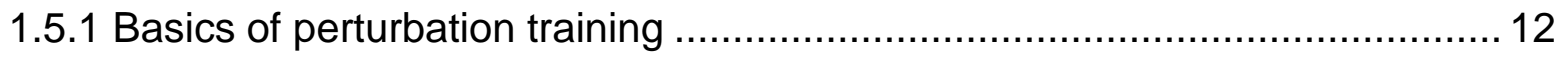

1.5.2 Methodologies of treadmill perturbation training ................................. 14

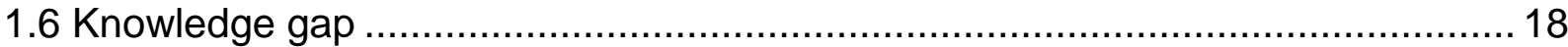

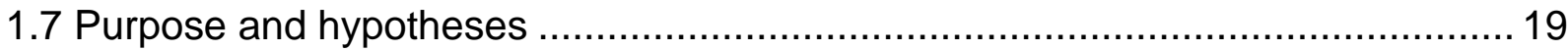

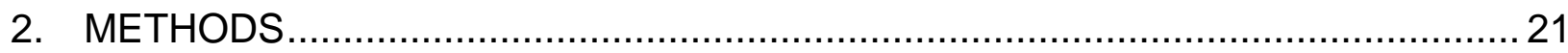

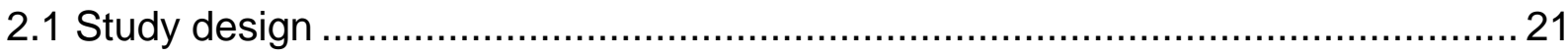

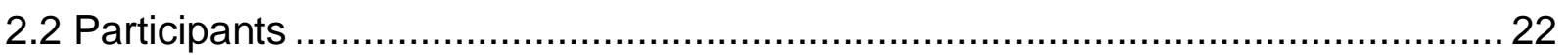

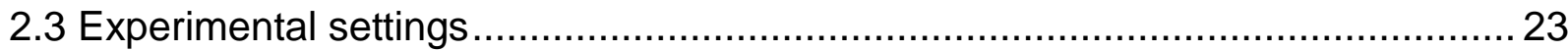

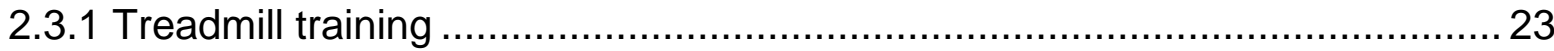

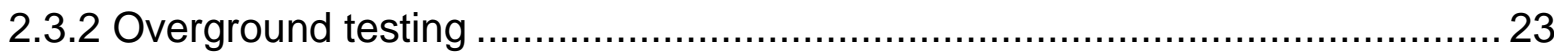

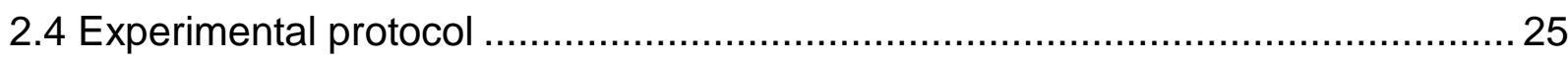

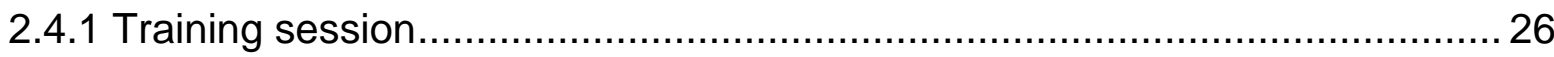

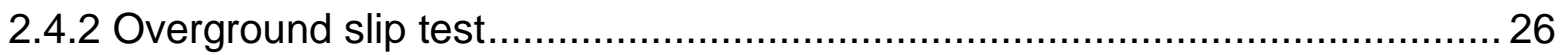




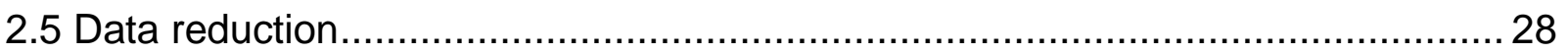

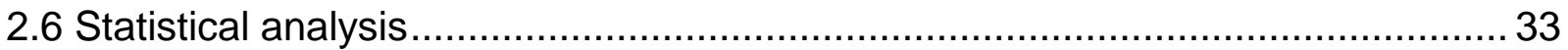

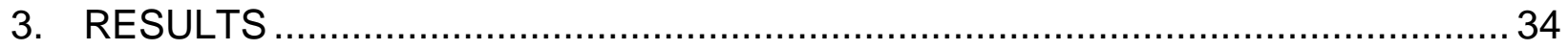

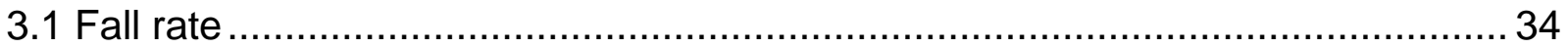

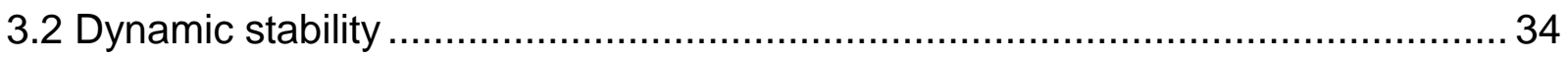

3.3 Temporal measurement and BOS kinematics.............................................. 35

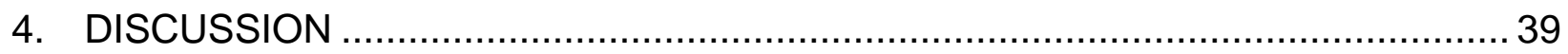

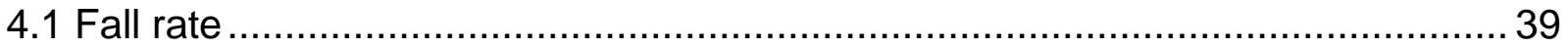

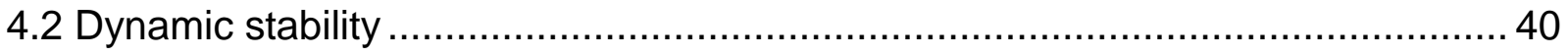

4.3 Temporal measurement and BOS kinematics ............................................. 43

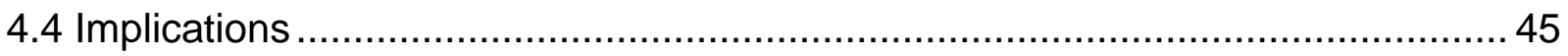

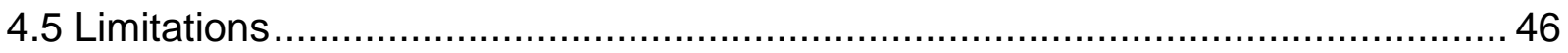

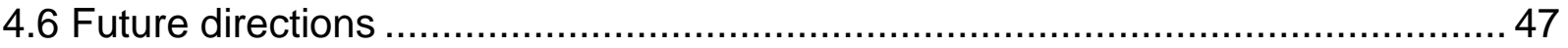

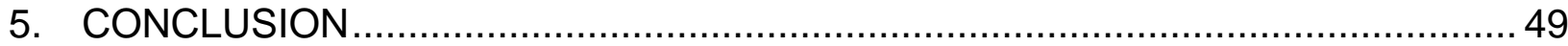

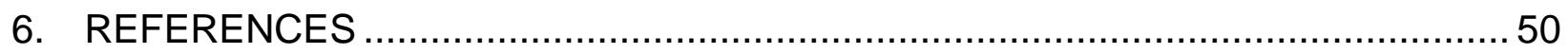

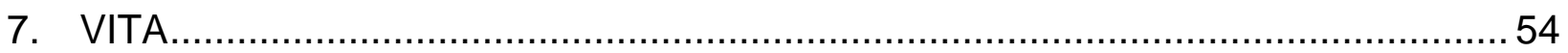




\section{LIST OF TABLES}

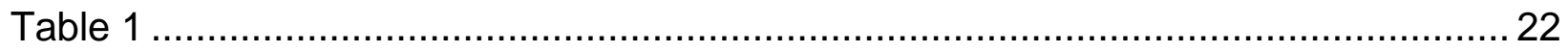




\section{LIST OF FIGURES}

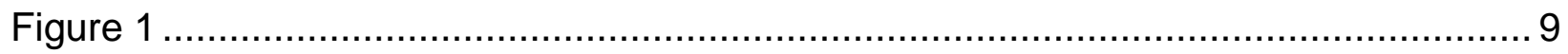

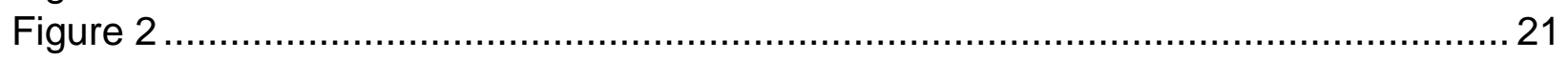

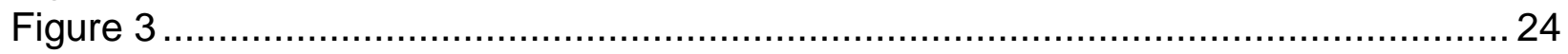

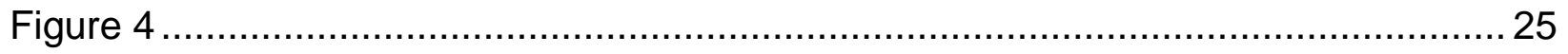

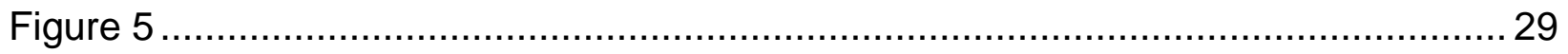

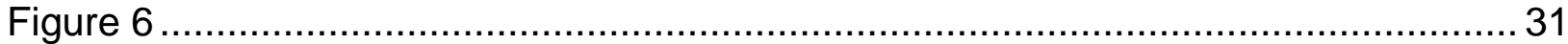

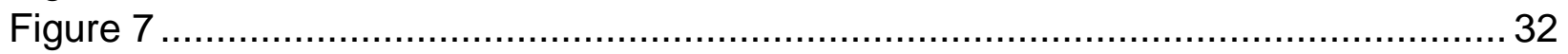

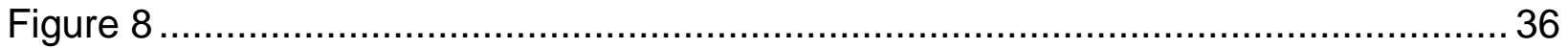

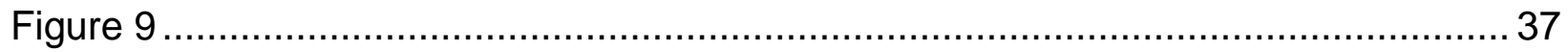

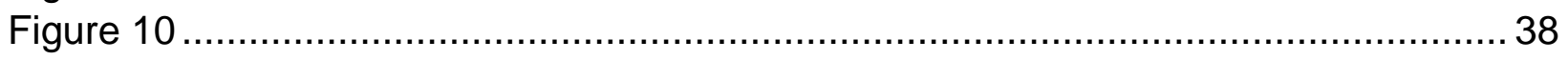




\section{INTRODUCTION}

\subsection{Falls in older and young adults}

Falls present a serious challenge among older adults, affecting not only the frail or impaired but the active and vigorous (Alexander, Rivara, \& Wolf, 1992). It is estimated that one out of every three older adults fall at least once a year (Stevens, Corso, Finkelstein, \& Miller, 2006). According to the Center for Disease Control and Prevention, the number of unintentional fall death rates have risen approximately $38 \%$ between 2004 and 2013 among adults aged 65 and older (Center for Disease Control and Prevention, 2015).

Falls also position older adults at a high risk of sustaining serious and lifethreatening injuries. It has been reported that approximately $20 \%$ of falls may result in a broken bone or serious head injury (Alexander et al., 1992). For instance, approximately $95 \%$ of hip fractures in the elderly are caused by a fall (Hayes et al., 1993). Falls are also responsible for most traumatic brain injuries among the elderly (Stevens et al., 2006). These types of injuries can have detrimental physical and emotional effects on the elderly and their families.

Although fall-related physical injuries can be avoided, in some cases among older adults, falls could cause the individual to become afraid of falling in the future, building up the fear of falling. Fear of falling consequently leads seniors to being physically inactive and weaker, thus increasing their likelihood of suffering from future falls (Vellas, Wayne, Romero, Baumgartner, \& Garry, 1997). It could also result in 
nursing home admittance, loss of independence, and greatly diminished quality of life (Stevens et al., 2006).

Along with physical and emotional detriments of sustaining a fall, there are also economic consequences that may occur to an individual as a result. Each year, over 200,000 older adults are hospitalized for hip injuries due to falls, which represents the most common injury sustained after a loss of balance (Parkkari, et al., 1999). Because a fall can cause serious physical harm, a vast portion of older adults who experience a fall must visit a hospital to ensure that they are treated for their injuries. Any type of stay at a hospital, whether it is short or extended, places these individuals in a great amount of economic stress. It has been reported that fall injuries lead to direct medical costs totaling to approximately $\$ 34$ billion annually in 2006 (Stevens et al., 2006). This cost is expected to reach $\$ 43.8$ billion by 2020 (Englander, Hodson, \& Terregrossa, 1996). Because much of the elderly is retired or unemployed, this added economic stress further imposes detrimental effects on health, well-being, and quality of life in this population.

While older adults are subject to high risk of falls and injuries, younger adults are also at a high risk of suffering from a fall-related injury. In a study by Heijnen \& Rietdyk, (2016), it was documented that $58 \%$ of the participants $(n=94)$ reported falling over the course of a 16-week semester at a university. The authors also reported that $16 \%$ of those who experienced a fall sustained an injury, and concluded that one fall occurred for every 18 slips or trips (Heijnen \& Rietdyk, 2016). This is mostly due to the unstable nature of human locomotion resulting from the invert pendulum structure of the human body and the high center of mass (COM) along with the small base of support (BOS). 
Those who work in conditions exposed to high fall hazards, also face an elevated risk of falls and related injuries. These conditions are predominately hazardous in themselves (construction work, factory work, etc.) because they require individuals to perform tasks that are inherently dangerous while using heavy machinery that could cause severe injuries or death, should an accident occur. Falls and injuries, constituting more than $10 \%$ of all work-related injuries, cause serious physical and medical consequences in young adults working in dangerous occupational conditions (Lanier, Baker, \& Sum, 2015).

The consequences of falls in young adults are like those of older adults. Falls can also leave young adults physically harmed, which may lead to psychological and economical stress (Lanier et al., 2015). Most injuries from a fall occur while a young adult is working with heavy machinery, in a hazardous job, or while taking part in an activity that compromises their locomotion. These injuries are more detrimental because of the environments that they take place (Heijnen \& Rietdyk, 2016).

Given the costly medical, healthy, psychological, and socioeconomic consequences resulting from falls, it is of immense importance to develop interventions to prevent falls from happening among both young and older adults. However, because the risks that come from sustaining a fall are more severe in older adults, the majority of current literature covers mostly older adults. Therefore, most of the information covered in the current study is regarding older adults because of their high injury death rate in accordance to falls and the psychological and economic consequences of suffering an injury from a fall. 


\subsection{Slip-related falls}

While any type of fall could result in injuries, slip-related falls have been shown to be the most dangerous and common type of falls. Slip-related falls account for approximately $40 \%$ of fall experienced by the elderly while walking outdoors (Luukinen, et al., 2000). A slip-related fall is characterized as a fall where an individual experiences a slip, with their body falling backwards relative to their feet, causing their COM to shift behind their BOS to a point where a backward loss of balance occurs (Yang, Anderson, \& Pai, 2007). If the loss of balance cannot be stopped or reversed rapidly, a backward or sideway fall will be materialized depending upon the direction of the slip perturbation (Bhatt, Wening, \& Pai, Adaptive control of gait stability in reducing slip-related backward loss of balance, 2006). If an individual experiences a perturbation that causes their COM to shift anteriorly to her/his BOS, s/he would experience a forward balance loss. Similarly, it would develop into a forward fall (or a trip-induced fall) if the balance loss cannot be halted quickly, according to Yang, Anderson, \& Pai (2007).

Slip-related falls can be very dangerous for the older population because they can lead to a high hip fracture rate (Stevens et al., 2006). Along with an increased risk of hip fracture, slip-related falls can also cause head trauma and other skeletal injuries. Because slip-related falls mostly occur in the outdoor environment (in which a slippery surface or an unstable object exists), individuals who have experienced a slip-related fall may not receive immediate treatment. If the slip-related fall leads to a serious injury, the individual may not be able to contact emergency services or have the ability to transport themselves to a place where they could receive medical attention for their 
injuries. Therefore, the consequences of slip-related falls are even more serious than other types of falls as they lead to statistically more injuries (Luukinen et al., 2000). Work-related backward falls preceded by a slip are also particularly dangerous to young adults because they frequently lead to brain and hip injuries, alike those among older adults (Lehtola, Becker, \& Brown, 2000).

\subsection{Risk factors of falls}

To develop effective slip-related fall prevention interventions, the first and probably the most important step is to identify the factors which contribute to falls in older adults. Previous studies have spent considerable amount of efforts in this direction. Various factors, such as the muscle weakness, balance impairments, stability disturbance, sensation loss, medical usage, visual impairments, and fear of falling have been related to falls in older adults (Rubenstein, 2006; Moreland, Richardson, Goldsmith, \& Clase, 2004; Horlings, van Engelen, Allum, \& Bloem, 2008). The factors which have been closely related to slip-related falls are reviewed below.

\subsubsection{Muscle weakness}

As one ages, s/he experiences sarcopenia, which appears as the loss of muscle tissue due to the natural process of aging. Because of the loss of muscle tissue, sarcopenia then leads to loss of muscular strength and power due to the loss of overall muscle mass. As previously referenced, muscle weakness, particularly in the lower extremity, is one of the primary causes of slip-related falls among older adults (Rubenstein, 2006). This notion has been confirmed by Moreland, Richardson, Goldsmith, \& Clase, (2004) reporting that when compared to upper extremity weakness, lower extremity weakness is significantly related to falls among older adults. 
The linkage between muscle weakness and slip-related falls could be explained by the necessary body reactions after a postural perturbation during gait. Following a slip, to regain body balance and prevent an actual fall, one must generate quick and sufficient corrective reactions during the recovery stepping (Cham \& Redfern, 2001; Yang, Bhatt, \& Pai, 2009). Two major reactions to a slip perturbation have been identified. One reaction is a knee flexor moment which has the potential to drag the BOS toward the body's COM. Such a reaction improves the stability and increases the chance of successfully recovering from the slip perturbation (Cham \& Redfern, 2001; Yang \& Pai 2010). Another reactive response is the knee extensor moment from the recovery leg providing sufficient antigravity support to prevent a limb collapse (Cham \& Redfern 2001). Adequate lower extremity muscle strength is demanded to execute both reaction strategies. If one can produce great muscle strength, the chance to retard and even reverse the falling after the slip would increase, reducing the probability of falls. The importance of sufficient lower extremity muscle strength and power in preventing slip-initiated falls has also been demonstrated by studies conducted on a slip-inducing treadmill (Ding \& Yang, 2016; Han \& Yang, 2015).

\subsubsection{Body balance}

Another factor that puts older adults at heightened risk of experiencing a fall is the impaired body balance skill. While the balance disturbance could be related to muscle weakness, it is usually considered an independent key marker in identifying individuals who are at high risk of falling. It has been shown that a weak body balance could predict slip-related falls among older adults (Horlings et al.,2008). Research has shown that control of balance while the elderly is in a narrow base of support is crucial 
in determining whether or not an older adult would experience a prospective fall. Specifically, when their mediolateral sway on increases, while they are in a narrow stance, the more susceptible they are to experiencing a fall (Melzer, Benjuya, \& Kaplanski, 2004). When an individual is standing upright, once perturbed, the body will make adjustments to counteract that perturbation and remain upright. This is done by making postural adjustments or by taking a step with either foot to maintain balance (Horak \& Nashner, 1986). Most times, older adults are unable to react appropriately to these perturbations, causing them experience a fall (Kuo, 1995).

\subsubsection{Dynamic stability}

Stability during human locomotion is defined as the ability to restore or maintain the upright posture without altering the existing BOS when confronting with an internal or external disturbance to the movement. During gait, the body is constantly in a state of loss of balance with each footstep preventing a forward fall (Winter, Patla, \& Frank, 1990). In order to achieve this goal, each footstep must be placed correctly to control the momentum, velocity, and position of the body's COM (Pratt \& Tedrake, 2006;

Townsend, 1985). In order to understand what leads an individual to fall during gait, it is important to understand the relationship between a body's COM and BOS since falls indeed are a disruption of the kinematic relationship between COM and BOS during human gait.

As aforementioned, an individual experiences a fall when their COM moves away from their BOS and such disruption breaks a certain threshold. A theoretical framework (the feasible stability region theory, or FSR) that extends the concept of static stability (Borelli, 1680) to dynamic conditions (Hof, Gazendam, \& Sinke, 2005; Yang et al., 2007) 
suggests that dynamic stability can be characterized by the kinematic relationship between the body's COM motion state (i.e., the combination of COM position and velocity related to the BOS) and the analytically-derived stability limits (Fig. 1). Dynamic gait stability, measured based on this theoretical framework, has been identified as a key factor leading to slip-related falls (Yang et al., 2009).

During a slip, older adults are unable to control their BOS or their COM due to poor body balance and muscle weakness (Horlings et al.,2008; Rubenstein, 2006). When an older adults experiences a slip, there is a high possibility that their COM motion state will be below the FSR threshold and they may experience a backward loss of balance (Yang et al., 2013). 


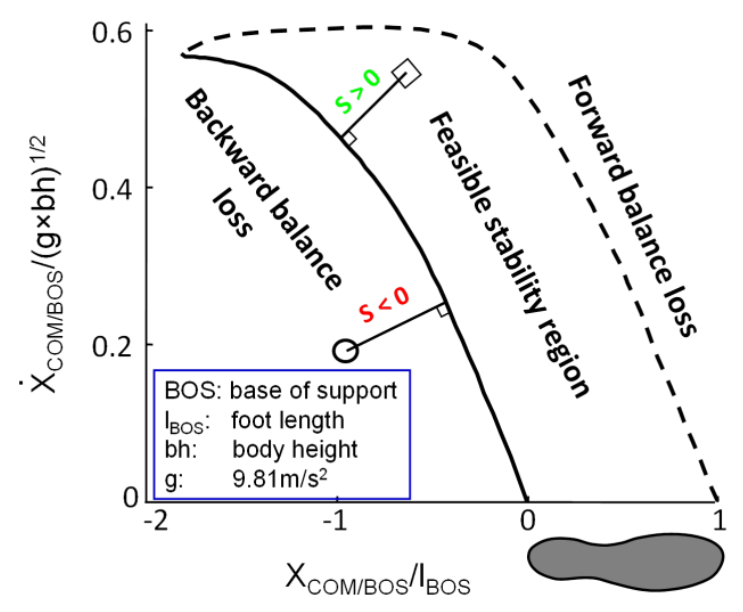

\section{Figure 1}

The feasible stability region (FSR) (Pai \& Patton, 1997). The FSR describes the region that the center of mass (COM) motion state (i. e, the combination of the COM velocity and position with respect to the base of support (BOS)) must stay within for an individual to maintain an upright body balance. The FSR consists of two boundaries. The lower one (the thick solid line) is called the limit against backward falling while the upper one (the dashed solid line) is the limit against forward falling. Dynamic gait stability $(s)$ is calculated as the shortest distance from the given COM motions state to the limit against backward falling, as indicated by the thin solid line. If the COM motion state is below the limit against backward falling, the dynamic stability is negative meaning that the individual has to take a backward recovery step to keep the body from falling backward, experiencing a backward balance loss. If the backward balance loss cannot be rapidly stopped, a backward fall would occur. Conversely, if the COM motion state is above the limit against forward falling, then the individual is forward instable. S/he must execute a forward recovery step to refrain the body from falling forward, leading to a forward loss of balance or an actual forward fall. When the COM motion state is within the FSR, the stability is a positive value indicating a stable state. Position and velocity of the COM relative to the BOS are dimensionless as a fraction of $l_{\mathrm{BOS}}$ and $\sqrt{g \times b h}$, respectively, where $l_{\mathrm{BOs}}$ represents the foot 
length, $g$ is gravitational acceleration, and $b h$ the body height.

\subsection{Falls prevention}

In the past decades, a variety of types of interventions have been developed to prevent falls in older adults mainly aiming at improving the risk factors of falls. Based on past research, these interventions have shown different levels of successes because the elderly are not as receptive to all interventions as a younger adult would be (Benichou \& Lord, 2016; Kummel, Kramer, Giboin, \& Gruber, 2016; Yang, King, Dillon, \& Su, 2015).

\subsubsection{Resistance or aerobic training}

Given the crucial role of muscle strength and power in preventing falls, resistance training has been applied to strengthen older adults. Progressive resistance training has been shown to increase muscle strength and balance in the elderly. Aerobic training has also demonstrated the similar effects in improving muscle strength or fitness in older adults, further reducing their risk of falls. However, this type of training modality has not been widely implemented (Benichou \& Lord, 2016). This is due to the fact that most seniors are unable to complete a progressive resistance training program because they are too weak or frail to even begin the program. Other factors limiting the implementation of this type training include the cost, location, and scheduling conflict, etc. (Benichou \& Lord, 2016). Thus, alternative methods for preventing falls which can be easily applicable to the elderly are highly desired. 


\subsubsection{Balance training}

Balance training is another effective intervention adopted to prevent falls among older adults. This type of training involves subjecting an individual to a series of movements on an unsteady surface. The theory behind this type of training modality is that the auxiliary muscle groups will aid the main muscle groups in maintaining stability. Balance training is popular among physical therapists and strength coaches, as it prepares an individual to meet the demands placed on them by their job, sport, or everyday life (Kummel et al., 2016).

While this form of training has proven to be affective, it is not easily and fully applicable to the older population because of the physical demands involved. If an older individual does not have enough muscle strength to maintain their stability while performing normal movements, it could become hard for them to perform specified balance exercises, as they may experience actual falls due to their high risk of falls. Another potential limiting factor is the long training duration it may require (Kummel et al., 2016).

Provided the limitations of resistance and balance training approaches, some easy yet effective training modalities are needed to reduce falls in older adults, which is the main purpose of this study. The ability to identify more accessible training interventions is crucial in preventing falls among the young and old alike.

\subsubsection{Vibration training}

Controlled whole-body vibration (CWBV) training has emerged as a relatively novel approach to prevent falls in older adults. This training involves the use of a 
vibration platform. This device requires an individual to stand on a platform while it mechanically vibrates and oscillates up and down. The mechanical stimulation transferred to the human body would induce neuromuscular reactions, in turn reducing the risk of falls in older population (Yang et al., 2015).

A study by Yang et al., 2015 showed that CWBV training improved muscle strength, joint flexibility, and lowered the fear of falling among the participants in the study. While this training method is promising because it is an easy training protocol for the elderly to take part in, there are a few pitfalls with it. For instance, vibration training usually requires a long-term training duration ( $4-50$ weeks) before solid training effects appear (Yang et al., 2015). This might limit its application to older adults. Because the interventions mentioned are not completely applicable to the elderly. This creates drawbacks from the current training interventions. To overcome the drawbacks of exercise-based and CWBV training approaches, a new training method - perturbation training - has recently been applied to fall prevention in older adults (Bieryla \& Madigan, 2011).

\subsection{Perturbation training}

\subsubsection{Basics of perturbation training}

Traditional training methods, such as strength training, could increase stability and balance in older adults after a slip occurs. However, research has argued that traditional methods are not specific enough to improve the neuromuscular reactions and adaptations in older adults (Granacher, Muehlbauer, Zahner, Golhofer, \& Kressig,

2011). Recently, a model has merged that relies on perturbation training to reduce the 
incidence of falls in older adults (Mansfield, Peters, Liu, \& Maki, 2010). Perturbation training focuses on forcing individuals to adapt to a perturbed environment and gain necessary techniques or skills to resist a fall should it occur after an internal or external disturbance to human gait. A recent study has demonstrated that perturbation training could improve balance recovery among older adults when they experience a slip or trip during gait (Bieryla \& Madigan, 2011).

Perturbation training has been employed in both static and dynamic situations. For example, few recent studies concluded that perturbations given to a participant on a motorized platform while standing in a static position improves overall body balance of the older individuals (Bieryla \& Madigan, 2011, Maki et al., 2008). Other studies attempted to exert the perturbation to human gait and demonstrated that such a perturbation paradigm can improve the stability of adults while they are walking. It was shown that dynamic gait perturbation training enables the central nervous system to effectively take actions to resist falls. These actions consist of the detection of an external perturbation and proactive adjustments in the body posture in order to maintain stability following the perturbation during gait. Another study examined the effect of a perturbation training protocol based on a special treadmill on reducing slip-related falls in young adults (Yang, Bhatt, \& Pai, 2013). The results revealed that treadmill-based perturbation training would in fact improve the dynamic stability and reduce falls among these adults as they experience an overground slip after the training.

Perturbation training is based on physical movement and learning. During the development of perturbation training paradigms, like treadmill-based perturbation training, researchers questioned whether or not observational training (i.e. visual and 
auditory training) could also improve dynamics stability among those who experienced an overground slip. This was an important question to answer because if an individual can learn to react properly to a overground slip without physical training, then it could undermine perturbation training as a whole. A study by Bhatt \& Pai (2008) compared the reactions of two groups to an overground slip: control (those who did not receive any type of training) and observational (those who received observational or verbal training). Participants in the observational group either watched pictures captured previously demonstrating individuals during a slip or received verbal training, where the researchers verbally taught the individuals how to deal with the overground slip. After the first overground slip, the control group completed a 24 repeated-slip training program. The response to the overgrond slip was compared between the two groups. It was shown that no difference in the response to the first overground slip was detected between groups. However, the control group exhibited significantly improved stability in comparison with the observational group post the repeated-slip training. It was concluded that the observational or verbal training may not be enough to induce improvement in stability responding to an overground slip. Actual and physical perturbation training, like treadmill-based perturbation training, is necessary to result in meaningful increase in stability in order to prevent slip-related falls.

\subsubsection{Methodologies of treadmill perturbation training}

The studies examining the effect of perturbation training in reducing falls among older adults are highly sparse. The most popular approach employed to conduct perturbation training is the use of special treadmills. One of these treadmills is the ActiveStep (Simbex, NH) treadmill. This treadmill is designed in a way that it can 
purposefully and suddenly change the moving direction of the belt speed to create an unexpected slip perturbation (Yang et al., 2013). Current applications for treadmillbased perturbation training cover a range of perturbations that occur in everyday life. These methods include standing-slip perturbations, in which the treadmill belt is suddenly moving while the participant is standing on the belt. Depending on the direction one faces during the experiment, the participant could be perturbed either on the anteroposterior or mediolateral directions (Mansfield et al., 2010). For example, Maki et al., (2008) carried out a study where the participants were asked to stand still on a moveable platform (alike a treadmill in this case) and the platform was unexpectedly moved to various direction. As a reaction to such a perturbation, individuals changed their BOS to reestablish the balance. After repeated perturbations, all individuals could proactively adjust their body posture, placing them in an advantageous position against possible falls resulting from the perturbation. The authors concluded that balance perturbation training could be a feasible method to reduce falls in older adults. However, the authors did not impose the perturbation on human gait. Thus, more comprehensive studies along this research direction are needed.

A study conducted through the use of a special perturbation treadmill, imposed the treadmill-based perturbation on human gait (Shimada, Obuchi, Furuna, \& Suzuki, 2004). Thirty-two older adults from a nursing home were enrolled in the study and assigned to the normal exercise group, in which they performed normal exercise as prescribed by the nursing home, or the treadmill exercise group, in which they received treadmill-perturbation training. The investigators found that, in relation to the normal exercise group, the treadmill exercise group had a significant improvement in balance 
and reaction time. Although these results showed that treadmill-perturbation training leads to better reaction times and balance among older adults, it could have been the based on the fact that their treadmill training was vastly different from their normal training.

Another study that investigated treadmill-based perturbation training looked into inducing a trip-like response during gait (Sessoms, Wyatt, Grabiner, Collins, Kingsbury, Thesing, \& Kaufman, 2014). Twelve male participants who had a recent lower limb amputation (with a prosthetic) were enrolled in the study. All participants underwent the perturbation training in which they walked on a treadmill and were suddenly exposed to a trip-like response on the treadmill. All participants experienced a fall when the perturbation occurred on the prosthetic side limb. After the first fall, all participants were able to successfully recover once when further perturbations were introduced to the participants. While this study proved that treadmill-perturbation training can allow participants with a recent amputation to adjust to the perturbations over time, the sample size was small and the population may have been the key factor to have led to the falls, and not the perturbation. Specifically, because the participants in this study had a recent leg amputation, the fact that they did not have a substantial amount of experience with their prosthetic may have been the reason that the individuals fell on the treadmill and not because of the perturbation induced by the treadmill. However, this study does provide valuable information about the validity of treadmill-perturbation training.

A recent pilot study inspected how and to what extent a treadmill-based perturbation training protocol could reduce slip-related falls among young adults. Thirty- 
four healthy young adults were enrolled and randomized into two groups. One group ( $n$ $=17$ ) underwent a single treadmill training session which was comprised of up to 30 slip trials. The other group $(n=17)$ did not receive this type of training. However, both groups were exposed to an identical overground slip. The results indicated that those who received the perturbation training fell much less than those who did not undergo the treadmill training. Similarly, the training group was more stable during the overground slip in comparison with the control group. One of the key reasons was that individuals in the training group knew how to proactively adjust the body posture prior to the slip during gait. Such adjustments improved the body stability level and reduced the likelihood of slip-related falls (Yang et al., 2013).

While past studies have proven to a certain extent that treadmill-based perturbation training can improve stability and prevent falls among the elderly, amputees, and young adults, it is also important to determine what treadmill intensity is most likely to prevent falls. A recent study investigated four different treadmillperturbation training intensities: high, low, progressively-increasing, and progressivelydecreasing (Liu, Bhatt, \& Pai, 2016). Thirty-six young participants were enrolled in the study and were assigned to one of the four groups. Grouping was even at nine participants per group. Each group experienced a total of 24 treadmill-perturbation slips before experiencing an overground slip similar to Yang et al., (2013). The researchers found that all groups exhibited improved balance and stability during the overground slip and they also found that the high intensity group yielded the strongest generalization, followed by the progressively-increasing group, the progressively-decreasing group, and the low intensity group, in that order (Liu et al., 2016). 


\subsection{Knowledge gap}

Although a recent study has examined the possible impact of treadmill-based perturbation training on reducing fall risk, the training session consisted of up to 30 slips in that study (Yang et al., 2013). A question remains unanswered is if the 30 slips are really necessary. Would a reduced number of trials still be able to achieve the same training effect? This is not a trivial issue. If a less number of slip perturbation trials can reach the same effect as the 30 slips, it would save the limited fall-prevention resources and redirect to others who need such training program the most. As a result, more people with a high risk of falls could benefit from this study.

In addition, very few studies have been carried out to investigate the effectiveness of treadmill-based perturbation training on reducing slip-related falls. There have also been few studies that have sought to induce an overground slip-related fall, which would simulate a fall in real-life. Therefore, the overall effect and efficacy of treadmill-based perturbation training on reducing real-life slip-related falls still largely remains unknown. It is imperative to examine the effect of treadmill-based perturbation training in reduce falls. To be safe, we would start with young adults. Young adults are also at risk of sustaining an injury from a slip-related fall, however because they predominately stronger and have better balance and dynamic stability than older adults, it is crucial to examine the effect of a treadmill-based perturbation training intervention before we can test this intervention with older adults. If our finding is in favor of the effect of treadmill-based perturbation training in reducing falls, our next step would be to transform this training protocol to older adults. 


\subsection{Purpose and hypotheses}

The purpose of this study was to investigate the overall effect and feasibility of a treadmill-based perturbation training paradigm consisting of 8 slip perturbations on reducing slip-related falls among young adults. Specifically, this study examined if and to what extent the shortened perturbation training protocol can reduce the rate of falls and improve the dynamic stability in response to a real-life like overground slip after the perturbation training procedure.

Aligning with the objectives of the study, the following hypotheses were tested:

1) Treadmill-based perturbation training will reduce the rate of falls among healthy young adults;

2) Treadmill-based perturbation training will improve dynamic stability in response to an overground slip in healthy young adults.

As aforementioned, falls lead to drastic direct and indirect costs (Alexander et al., 1992). If our results support that a reduced perturbation-training protocol based on a commercialized treadmill can reduce the risk of falls among older adults and young workers who face working environment with fall hazards, this type of training can be clinically deployed to clinical settings, senior centers, or nursing homes. With the reduction of falls, the costs resulting from falls and relevant injuries will be lowered as well.

The long-term goal of this research line is to systematically examine the immediate and long-term effectiveness, efficacy, and safety of perturbation training. With those three aspects examined and proven, it is possible to seek insurance 
coverage for this type of training. By so doing, the training based on a treadmill could be applied as a "vaccine" to prevent or reduce future falls among older adults or young workers, which would make significant medical, healthy, socioeconomic, and psychology effects on our health care system. 


\section{METHODS}

\subsection{Study design}

This study adopted a randomized controlled trial (Fig. 2). Forty-three healthy young participants were randomly assigned into either the training or control group. The training group underwent a treadmill-based perturbation training protocol while the control group received a mocking training. The mocking training was conducted on the same treadmill and participants were asked to walk the same amount of time on the treadmill as the training group. After the training, both groups were exposed to an identical, unannounced overground slip. Their responses to this slip were recorded and compared between groups.

$\mathrm{R}=$ Randomization phase

$\mathrm{H}=$ Hypothesis

- $\mathrm{H}_{1}=$ fall rate at testing: training $<$ control

- $\mathrm{H}_{2}=$ dynamic stability at testing: training $>$ control

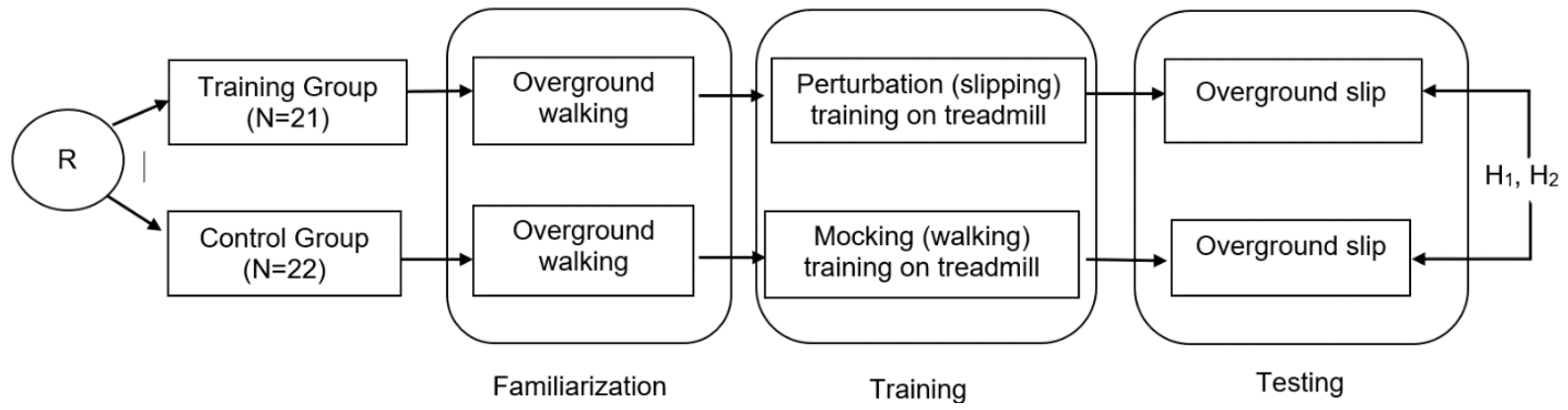

Figure 2

Schematics of the study design. 43 participants were randomized into two groups: training and control groups. After similar familiarization of the overground walking, the training group underwent the perturbation training (including 8 slips) on the treadmill while the control group only walked on the treadmill for the same number of trails without perturbation administered. Their fall rate and dynamic stability in response to an unexpected overground slip after the training was compared to testify the two hypotheses. 


\subsection{Participants}

Forty-three healthy young adults were recruited for this study from the university campus and throughout the El Paso region (Table 1). No restrictions were applied to the participants' gender and ethical background. To exclude some confounding factors which may affect our findings, such as medical condition, inclusion and exclusion criteria were established. Inclusion criteria for the participants were that participants must be between the 18 and 45 years old and no any neurological, muscular, or skeletal problems. Participants were excluded from the study if they are not within the age range listed and if they suffer from a physical or cognitive disease that prevents them from full mobility. Before the participation in the study, each participant provided a written consent form which was approved by the Institutional Review Board of the University of Texas at El Paso (IRB: 723376-3).

Table 1 Comparison of the demographic information in mean \pm standard deviation between the training and control groups.

\begin{tabular}{cccc}
\hline Parameter & Control $(n=22)$ & Training $(n=21)$ & $p$-value \\
\hline Age & $22.65 \pm 3.8$ & $22.57 \pm 3.2$ & 0.94 \\
Gender (female) & 8 & 14 & $0.12^{\star}$ \\
Height $(\mathrm{m})$ & $1.67 \pm 0.09$ & $1.70 \pm 0.08$ & 0.24 \\
Mass $(\mathrm{kg})$ & $71.14 \pm 13.36$ & $74.95 \pm 17.98$ & 0.44 \\
BMl $\left(\mathrm{kg} / \mathrm{m}^{2}\right)$ & $25.35 \pm 3.21$ & $25.62 \pm 4.41$ & 0.82 \\
\hline
\end{tabular}

BMI: the body mass index.

*: Fisher's exact test used. 


\subsection{Experimental settings}

\subsubsection{Treadmill training}

Both the perturbation and mocking training were conducted on the ActiveStep $\AA$ treadmill (Simbex, NH) (Fig. 3A). This specialized treadmill is able to produce a sudden acceleration to instantly alter the belt velocity (either the magnitude or the direction, or both) to induce a slip or trip perturbation (Fig. 3B). During all trials on the treadmill, each participant wore a full-body safety harness (Maine Anti-Gravity systems, ME) which was connected to an overhead arc through dynamic ropes. This system protected the participants from any possible injury if a fall occurred after a slip perturbation (Fig. 3A).

\subsubsection{Overground testing}

After the training (either perturbation training for the training group or the mocking training for the control group), all participants were exposed to an identical overground real-life like slip. This slip perturbation was induced by a customized sliding device which resided on the right side of the middle section of the linear walkway (Fig. 4A). This device consisted of a linear railway upon which two linear bearings can smoothly travel. The bearings were attached to an aluminum platform. During normal walking trials, the railway was covered by a wood plate with the same color as the walkway. The platform was at ground-level to reduce its visibility to the participants. The platform was firmly locked during normal walking trials by two pins so the participants could not feel the possible movement of the platform, reducing their prior knowledge about the impending slip. Immediately before the slip trial, the pins and the wood plate was removed without the participants' knowledge. During all trials, the lights were switched 
off to further lowering the possibility of participants guessing the locations of the slippery surface. When the participants stepped on the platform, his/her foot would travel forward with the platform for up to $1.0 \mathrm{~m}$, creating an unannounced, real-life like slip perturbation (Fig. 4A). Participants were protected by the full-body harness system which was connected to a ceiling-mounted I beam through dynamic ropes and a load cell (Transcell Technology Inc., IL) during the overground slip. The length of the ropes connected to the harness was standardized (Fig. 4B).

A. Treadmill

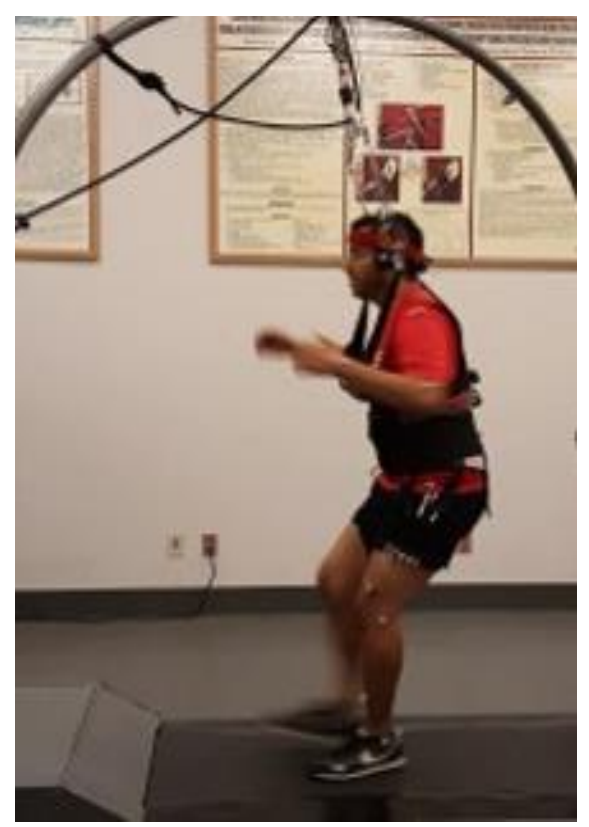

B. Belt Speed Profile

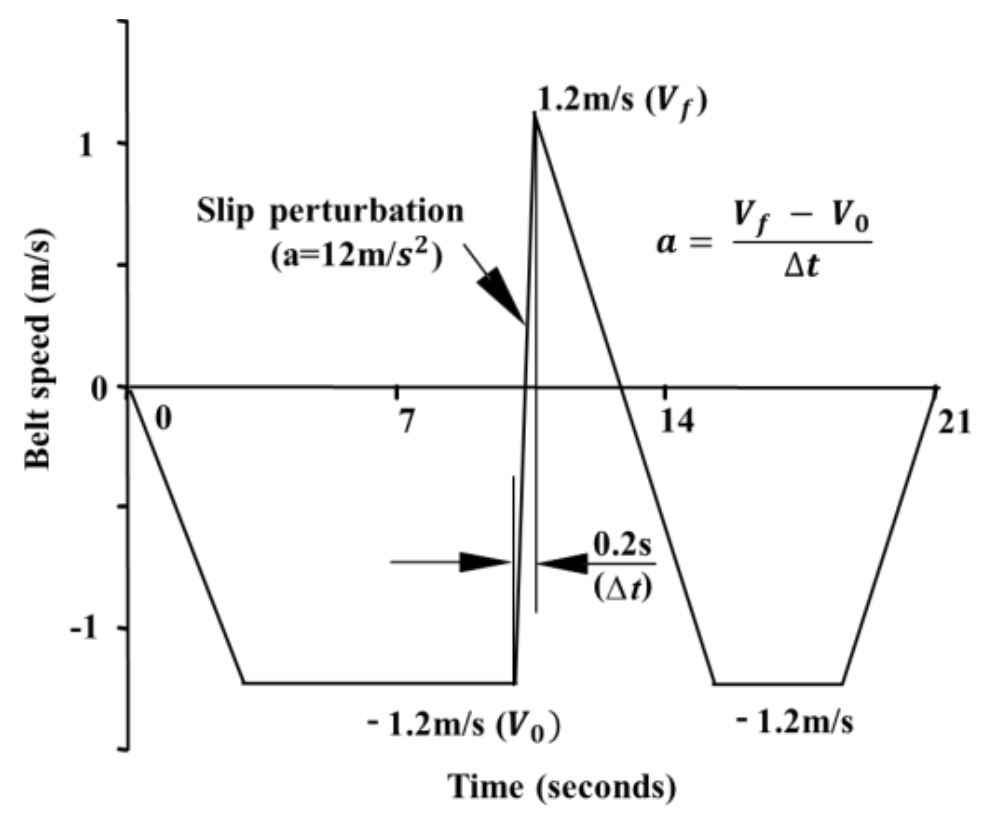

Figure 3

Picture A shows the treadmill (ActiveStep, $\mathrm{NH}$ ) used to induce a slip perturbation during walking under the protection of a harness system. Picture B shows the treadmill belt speed profile used to perturb the participants unexpectedly on the treadmill during training. The perturbation level was characterized with the acceleration of $12 \mathrm{~m} / \mathrm{s}^{2}$, peak slip velocity of $2.4 \mathrm{~m} / \mathrm{s}$, and the slip distance of $24 \mathrm{~cm}$. 


\subsection{Experimental protocol}

After demographic and anthropometric data has been collected, each participant had a session to become familiar with overground walking on the walkway. They were instructed to walk about 6 times along the walkway with the safety harness. Afterwards, they stepped on the treadmill undergo the training (either the perturbation or mocking training). Following the training session, they were brought back to the walkway. After few more normal walking trails, they experienced the overground slip.

A. Overground slip platform

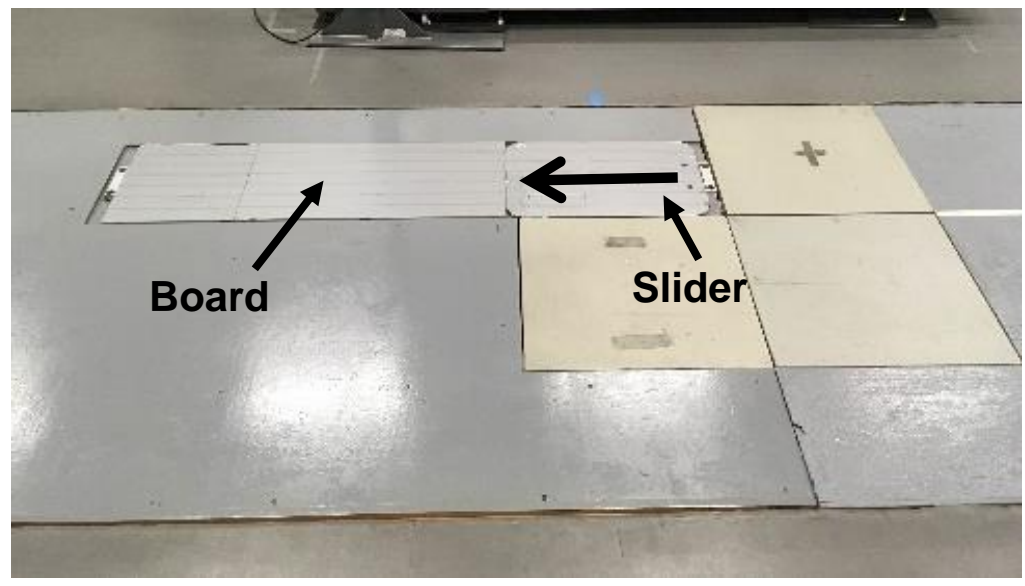

B. Harness adjustment

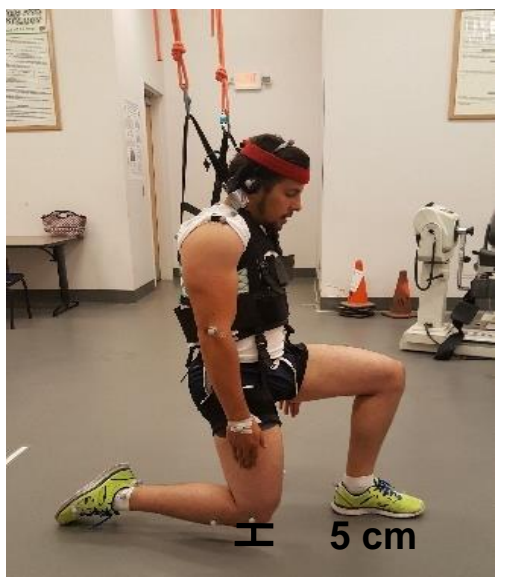

Figure 4

Picture A shows the overground platform used to induce slip perturbation during gait. Picture $B$ shows the overground harness system and the testing method used to ensure the safety of the participants during overground slip. Once secured, the participants were asked to lunge forward and to try to touch their knee to the ground. The harness is deemed safe once the participant's knees were approximately $5 \mathrm{~cm}$ above the ground. 


\subsubsection{Training session}

After the initial overground walking, all participants were moved to the treadmill. After approximately 6 normal walking at a speed of $1.2 \mathrm{~m} / \mathrm{s}$ each lasting about $30 \mathrm{~s}$, participants received their assigned training. For the training group, they underwent 5 slips followed by 3 normal trials and then 3 slips. The slip perturbation level was 12 $\mathrm{m} / \mathrm{s}^{2}$ of acceleration, $0.24 \mathrm{~m}$ of slip displacement, and $2.4 \mathrm{~m} / \mathrm{s}$ of peak slip velocity. Each slip trial began with a 2-s ramp up, followed by a steady state with a backward belt slip of $1.2 \mathrm{~m} / \mathrm{s}$ (Fig. 3B). After 10-12 regular steps, approximately $80-120 \mathrm{~ms}$ following the touchdown of the leading foot, the top belt suddenly accelerated forward within $0.2 \mathrm{~s}$ without the participants' knowledge. Following the slip perturbation, the top belt speed slowly returned to backward direction at $1.2 \mathrm{~m} / \mathrm{s}$. After the first slip, the participants were instructed that "what you just experienced was a slip trial. From next trial on, you may or may not be slipped on every trial. If a slip occurs, try your best to keep you from falling or losing balance, and continue walking forward. Try not to grab onto the ropes." The control group underwent the same number of trails on the treadmill without slip, which formed the mocking training protocol.

\subsubsection{Overground slip test}

Following the protocols on the treadmill, all participants were brought back to the overground walkway and the harness was connected to the ceiling-mounted system. The participants were instructed to face away from the walkway before each trial until they were told to turn around. When participants were standing, and facing away from the walkway, the lights in the room were turned on and the experimenter made noise in the middle of the walkway. Then, the lights were turned off and the participants were 
asked to turn around and face the walkway. They were asked to look straight forward and walk at their comfortable speed in their preferred manner. They were also clearly told that no slip perturbation would happen during the first three trials. After completing these three normal walking trials on the walkway, they were told that "from the next trial one, you may or may not be slipped on every trial. If a slip happens, try your best to recover your balance and keep walking forward. Try not to grab onto the ropes." After approximately 8 more regular walking trials in which the starting position was adjusted to make sure the participants would land their right foot in the middle section of the movable platform, the movable platform was released by removing the two pins and the wooden platform was removed. Because the dimmed lights in the room during all trials, it was extremely hard for the participants to identify any difference in the walkway. The overground slip test concluded the experiment.

The full-body kinematics of all walking trials on the treadmill or overground were recorded using an 8-camera motion capture system (Vicon, UK) at $120 \mathrm{~Hz}$ from 26 reflective markers. The specific positions of these markers were: the vertex of the head, the $\mathrm{C} 7$ cervical disc, ears, the scapula, sacrum, bilateral shoulders, elbows, wrists, greater trochanters, thighs, knees, tibias, ankles, heels, and toes (Yang et al, 2009) (Fig. 5). These reflective markers were used to create a 13-segment model for COM kinematic calculations (de Leva, 1996). A marker was also placed on the slider to register its movement. The load cell connecting to the ropes in series measured the force exerted on the ropes. The load cell force was recorded at $600 \mathrm{~Hz}$ and synchronized with the motion capture system and the video recording. 


\subsection{Data reduction}

The overground slip trial was chosen as the trial of interest. The force from the load cell was used to determine the outcomes (fall or recovery) of the overground slip trial. The slip trial was classified as a fall if the peak load cell force exceeds $30 \%$ body weight (bw) (Fig. 6A). A recovery occurred when the moving average of the load cell force on the harness did not exceed $4.5 \%$ bw over any 1 -second period after the slip onset (Fig. 6B). If the peak value of the moving average load cell force over any 1 second period exceeded $4.5 \% \mathrm{bw}$, but the load cell force never reached a peak of $30 \%$ bw, this trial would be considered harness assistance. The harness assistance trials would be excluded from further analysis due to the uncertainty of determining the slip outcome without the harness. No trial was identified as harness-assistance in this study (Yang \& Pai, 2011). Marker paths was low-pass filtered at marker-specific cut-off frequencies (ranging from 4.5 to $9 \mathrm{~Hz}$ ) using fourth-order, zero-lag Butterworth filters (Winter, 2005). Locations of joint centers, heels, and toes were computed from the filtered marker positions. 
A. Front view

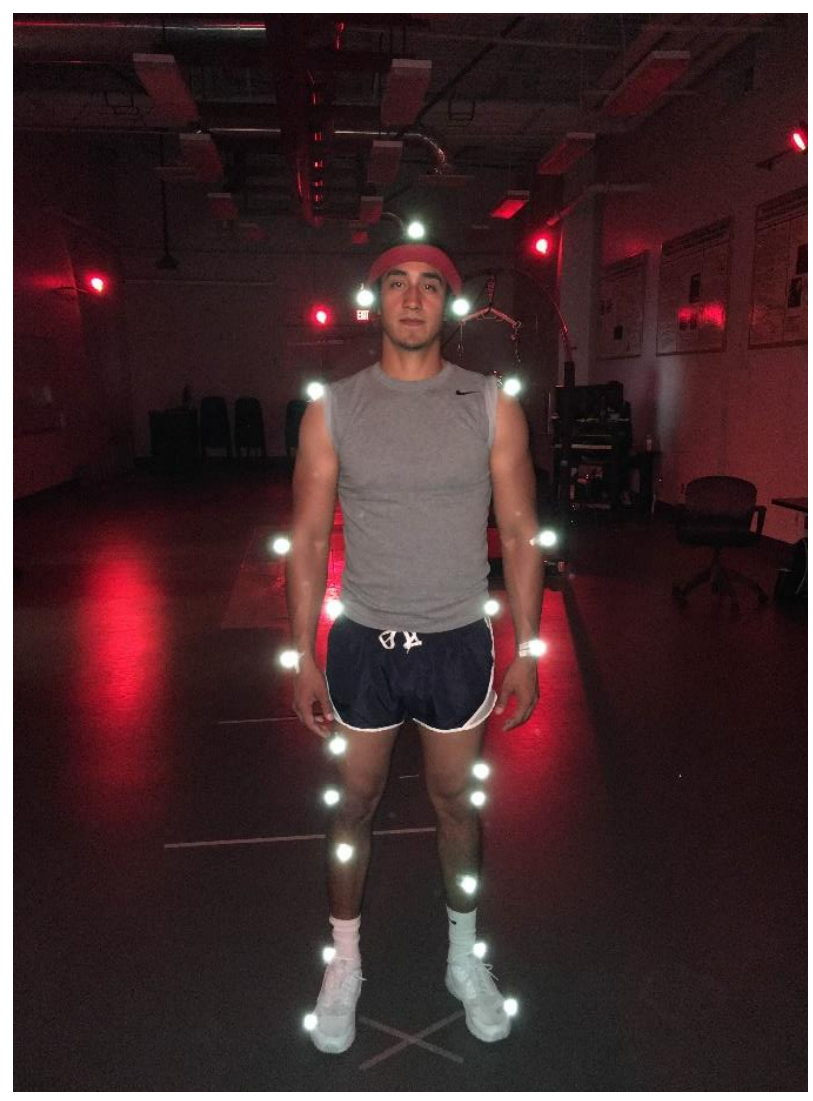

B. Side view

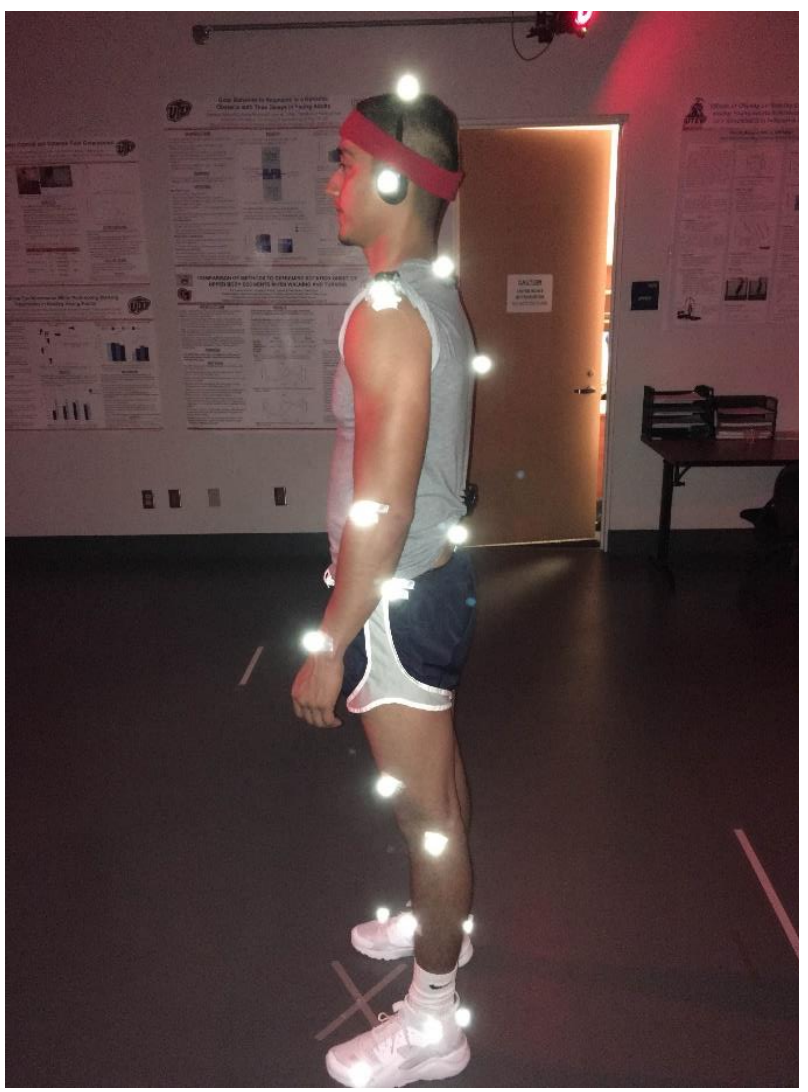

Figure 5

Demonstration of the locations of all 26 reflective makers attached to the participant during all walking trials on the treadmill or over ground from the $(A)$ front and $(B)$ side view.

Besides the slip outcome, three outcome measurements in the overground slip trial were examined: the COM position, COM velocity, and dynamic gait stability. They were calculated at three events: the touchdown (TD) of the right foot on the platform (RTD), the liftoff (LO) of the left foot after slip onset and its TD (LLO and LTD). These three events were identified based on the feet kinematics (Figs. 6C \&D). Specifically, the time of the TD was determined when the vertical velocity of the heel marker was below $0.05 \mathrm{~m} / \mathrm{s}$ while LO was the instant when the vertical velocity of the heel marker was greater than $0.05 \mathrm{~m} / \mathrm{s}$. 
The body COM displacement was computed using gender-dependent segmental inertial parameters (de Leva, 1996) and its velocity was calculated as the first order displacement with respect to time. The two components of the COM motion state, i.e. its position and velocity was calculated relative to the rear of BOS (i.e. the leading heel) and normalized by foot length (/BOS) and $\sqrt{g \times b h}$, respectively, where $g$ was the gravitational acceleration and $b h$ was the body height. Dynamic stability was calculated as the shortest distance from COM motion state to the limit of stability (Fig. 1) (Yang, Bhatt, \& Pai, 2009).

The duration of the double (from RTD to LLO) and single (from LLO to LTD) phases after the slip onset were also calculated. To inspect the movement of the BOS during the slip, the displacement and velocity of the BOS (i.e., the slider) on the anteroposterior direction were also determined. The forward velocity of the slider was calculated as the first order derivate of its anteroposterior displacement with respect to time. Both the BOS displacement and velocity were calculated at all three events (Fig. 7). 


\section{A. Load cell force - Fall}

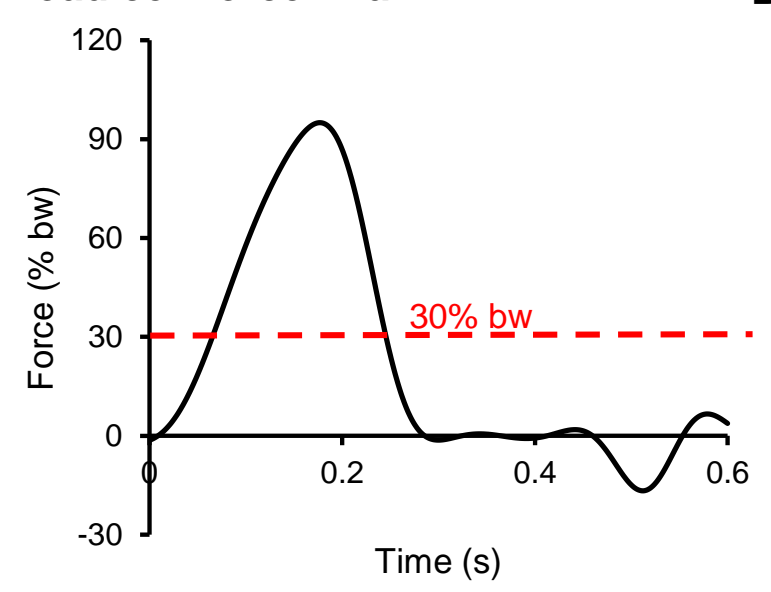

C. Fall

RTD

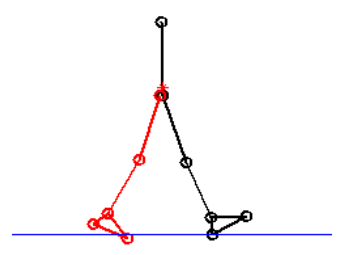

\section{Recovery}

RTD

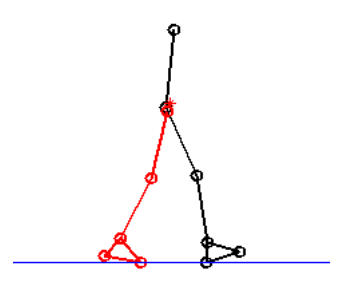

LLO

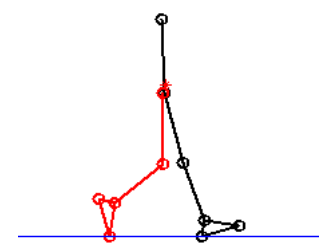

LLO

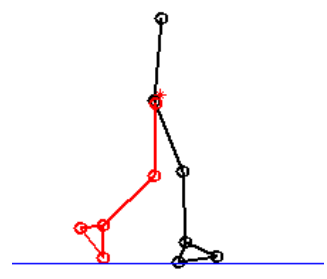

B. Load cell force - Recovery

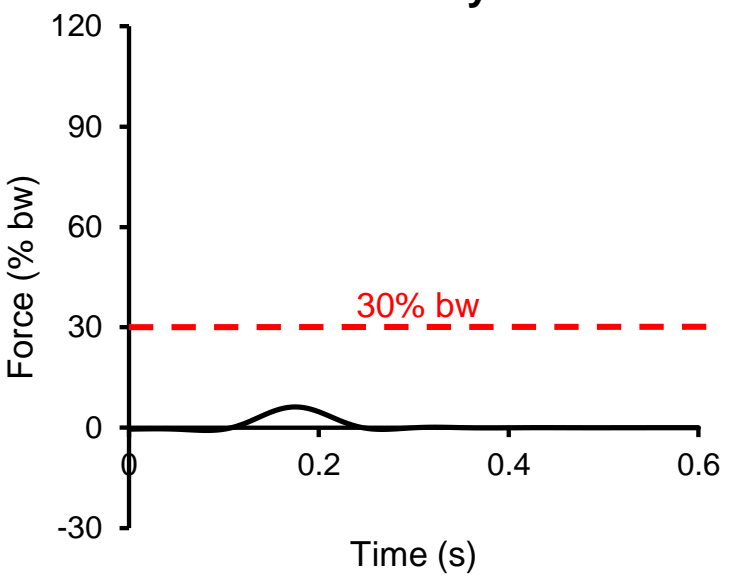

LTD

Fall
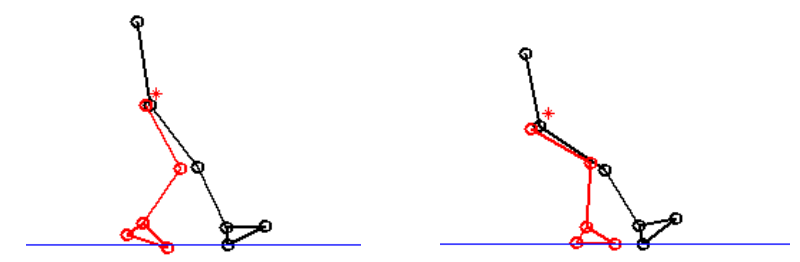

LTD

Recovery

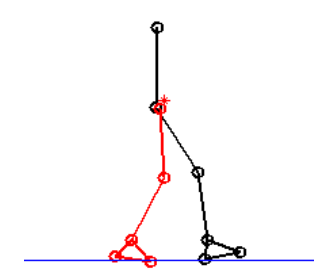

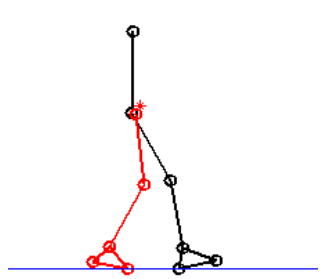

Figure 6

Depictions of the determination of time events during an overground slip trial. The gait events of interest included the right (or slipping) foot touchdown (RTD), left (or recovery) foot liftoff (LLO), and its touchdown (LTD) after the slip onset for (A) a fall and (B) a recovery trial. The trial was considered (C) a fall if the load cell force was $>30 \%$ of their body weight (bw) and (D) a recovery if the moving average of the load cell force on the harness did not exceed $4.5 \%$ bw over any 1 -second period after the slip onset. 


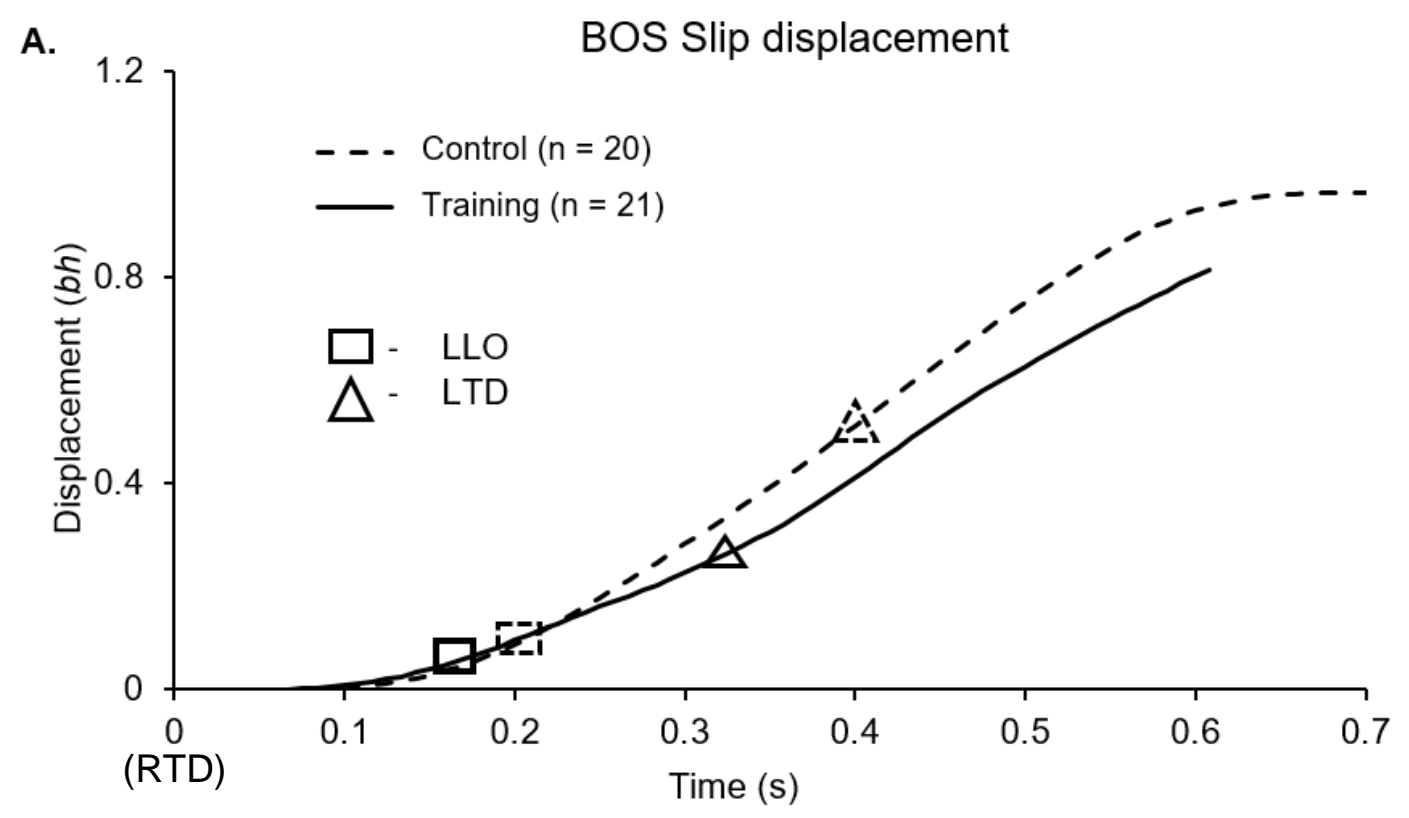

B.

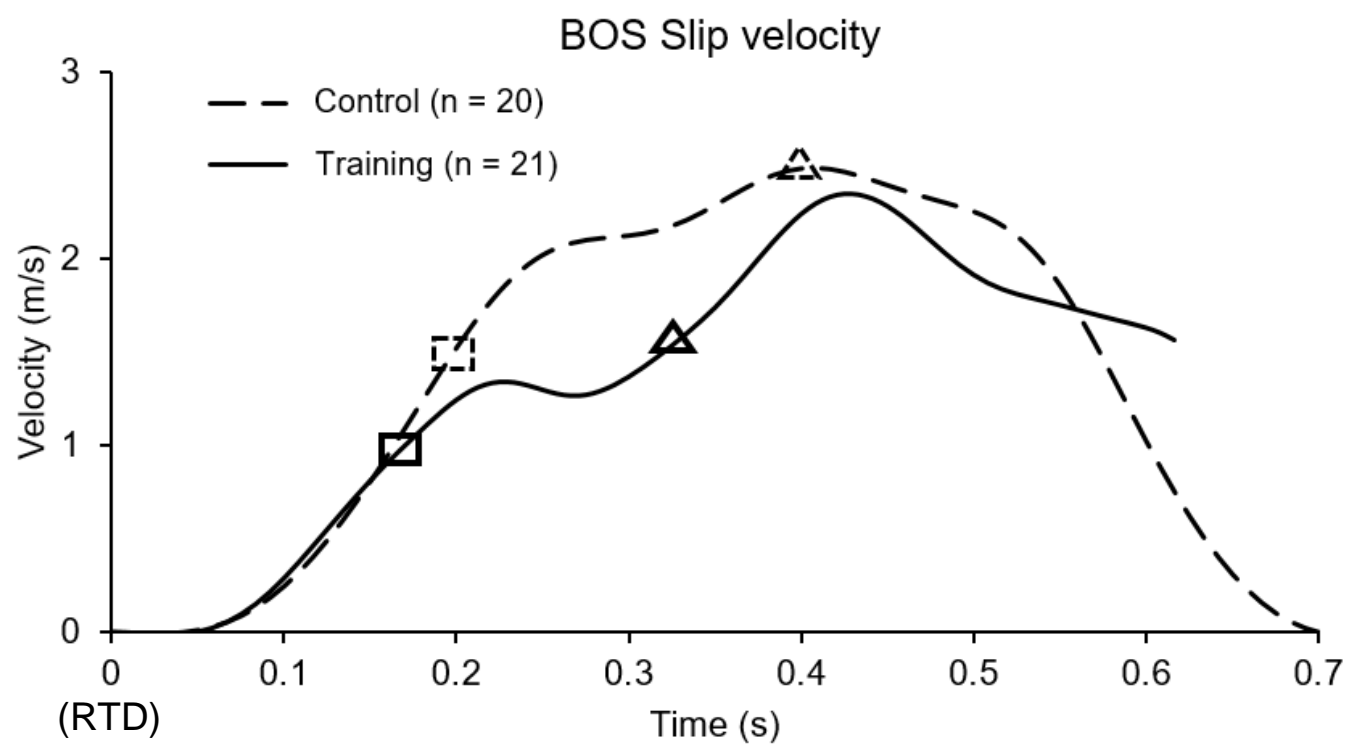

Figure 7

Representative time histories of $(A)$ the anteroposterior displacement and $(B)$ the forward velocity of the base of support (BOS) for both training and control groups. Time zero was the instant when the right foot was in contact with the slider (i.e., the right foot touchdown or RTD). Also shown are the instants of the left foot liftoff (LLO) and its touchdown (LTD). 


\subsection{Statistical analysis}

The body demographic information was firstly compared between groups to ensure the validity of the randomization procedures. A $X^{2}$ test was used to compare the fall rate in response to the overground slip between groups. The fall rate was calculated as the ratio of the number of fallers to the total number of participants in each group. Independent $t$-tests were applied to compare the spatiotemporal measurements including the duration of single and double phases, the COM position, velocity, and stability and the BOS kinematics between the two groups at all three events: RTD, LLO, and LTD. SPSS 21.0 (IBM, NY) was used for statistical analysis and a $p$-value $<0.05$ was considered significant. 


\section{RESULTS}

The demographic information was comparable between groups (Table 1), indicating the validity of our randomization procedure. All 43 participants completed the study protocol. However, the overground slip was not valid for two participants in the control group due to technical errors. Therefore, 20 and 21 participants respectively from the control and training groups were included in the final analysis.

\subsection{Fall rate}

In response to the same novel overground slip, all participants from both groups experienced a backward balance loss as indicated by taking a recovery step which landed posterior to the leading foot. 13 out of 20 (65\%) participants in the control group experienced a slip-related fall while only 4 out of $21\left(19.1 \%, p=0.003, X^{2}=8.912\right)$ fell when being exposed to the overground slip (Fig. 8).

\subsection{Dynamic stability}

At RTD, individuals in the training group demonstrated similar COM position relative to the $\mathrm{BOS}$ to the control group $(-1.099 \pm 0.169$ vs. $-1.147 \pm 0.232, p=0.448$, Fig. 9A). Similarly, both groups exhibited comparable COM velocity related to the BOS $(0.321 \pm 0.035$ vs. $0.315 \pm 0.055, p=0.687$, Fig. $9 \mathrm{~B})$ at the same instant. As a result, no significant difference was observed between groups with respect to dynamic stability at RTD $(-0.157 \pm 0.044$ vs. $-0.170 \pm 0.059, p=0.412$, Fig. 9 C $)$. The COM motion state and dynamic stability did not display significant difference between groups at LLO either (position: $-0.593 \pm 0.188$ vs. $-0.689 \pm 0.265, p=0.187$, Fig. 9 A; velocity: $0.073 \pm 0.102$ vs. $0.081 \pm 0.075, p=0.792$, Fig. $9 \mathrm{~B}$; and stability: $-0.233 \pm 0.126$ vs. $-0.257 \pm 0.107, p$ $=0.512$, Fig. 9C). 
At LTD, individuals in the training group significantly placed their COM more anterior (or closer) to the BOS compared to those in the control group $(-0.583 \pm 0.292$ vs. $-0.893 \pm 0.33, p=0.003$, Fig. $9 \mathrm{~A})$. Additionally, the training group demonstrated a significantly less backward COM velocity relative to the BOS than the control group ($0.095 \pm 0.080$ vs. $-0.178 \pm 0.092, p=0.004$, Fig. 9B). The training group was significantly less instable than the control group at LTD as evidenced by the significantly greater dynamic stability among the training group than in the control group $(-0.375 \pm$ 0.161 vs. $-0.566 \pm 0.160, p=0.001$, Fig. 9 C).

\subsection{Temporal measurement and BOS kinematics}

With respect to the temporal measurement, the training group was similar to the control group about the duration of the double stance phase from RTD to LLO $(0.154 \pm$ 0.046 vs. $0.134 \pm 0.046 \mathrm{sec}, p=0.175$, Fig. $10 \mathrm{~A})$. However, the training group exhibited a statistically shorter single stance phase (from LLO to LTD) in comparison with the control group $(0.145 \pm 0.068$ vs. $0.187 \pm 0.047 \mathrm{sec}, p=0.029$, Fig. $10 \mathrm{~A})$.

The BOS velocity (or the velocity of the slider) did not demonstrate any grouprelated difference $(1.128 \pm 0.475$ vs. $1.123 \pm 0.28 \mathrm{~m} / \mathrm{s}, p=0.968$, Fig. 10B); so did the BOS displacement $(0.036 \pm 0.017$ vs. $0.036 \pm 0.281 b h, p=0.881$, Fig. $10 \mathrm{C})$ at LLO. Whereas, the BOS travelled more slowly $(1.684 \pm 0.346$ vs. $1.919 \pm 0.388 \mathrm{~m} / \mathrm{s}, p=$ 0.046, Fig. 10B) with shorter distance $(0.153 \pm 0.032$ vs. $0.220 \pm 0.042 b h, p<0.01$ Fig. $10 C)$ at LTD in the training group than in the control. 


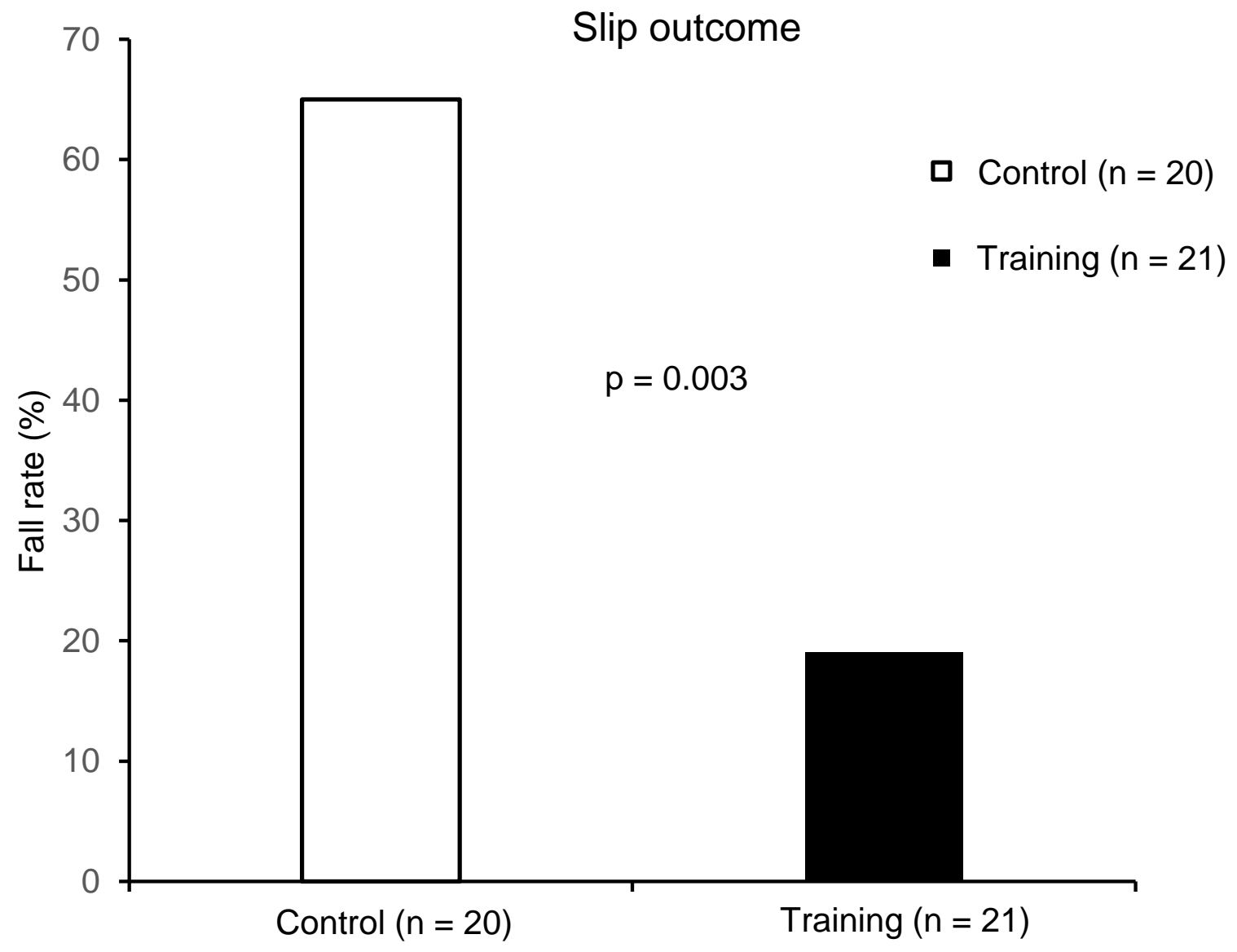

Figure 8

Comparison of incidence of falls in response to an unannounced novel overground slip between the training and control groups. The fall rate was calculated as the ratio of the number of fallers to the number of total participants in the same group. 
A.
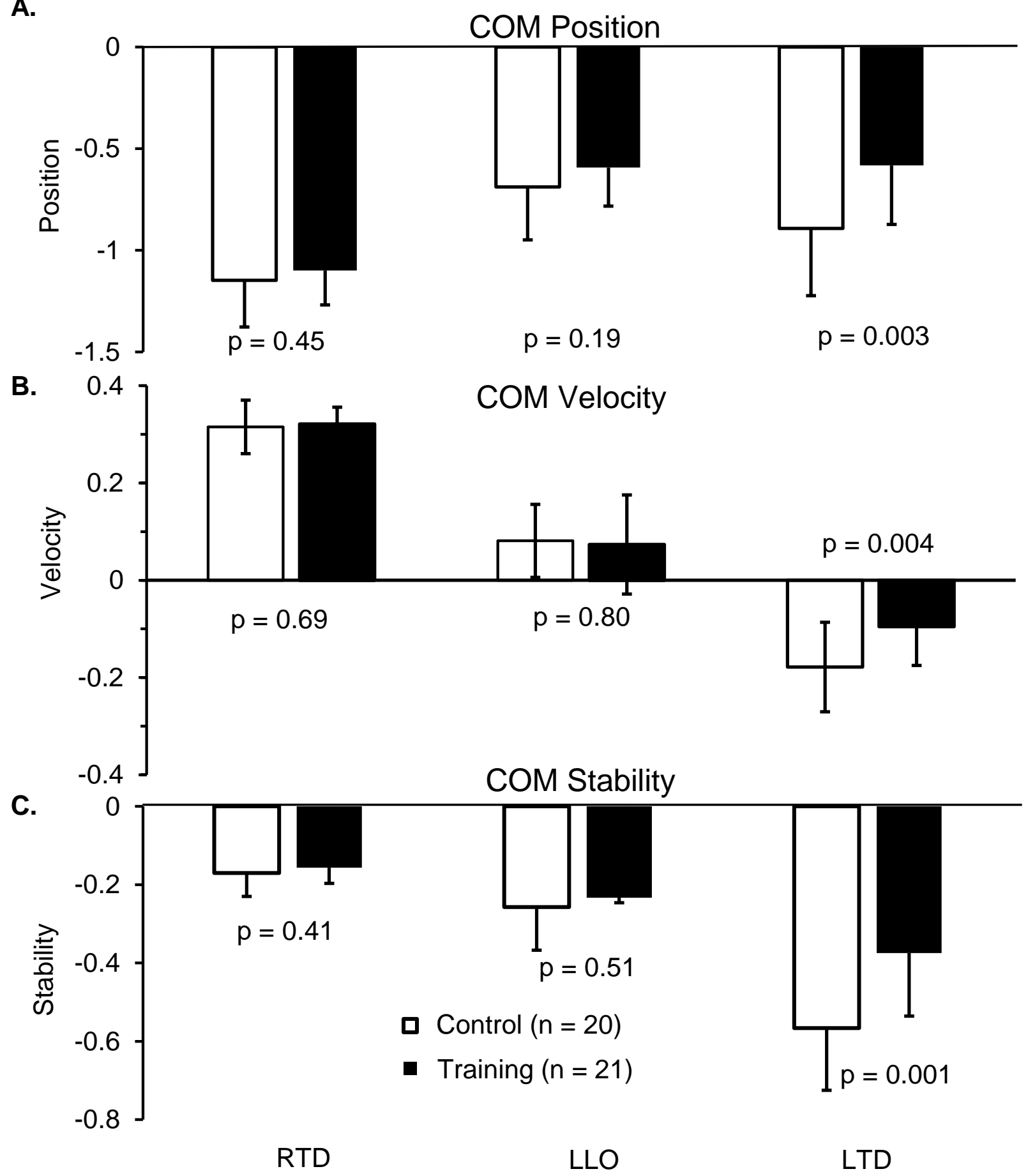

Figure 9

Comparisons of $(A)$ center of mass (COM) position, $(B)$ COM velocity and $(C)$ dynamic stability between the training and control groups at right foot touchdown (RTD), left foot liftoff (LLO), and left foot touchdown (LTD) during the overground slip. 
A.

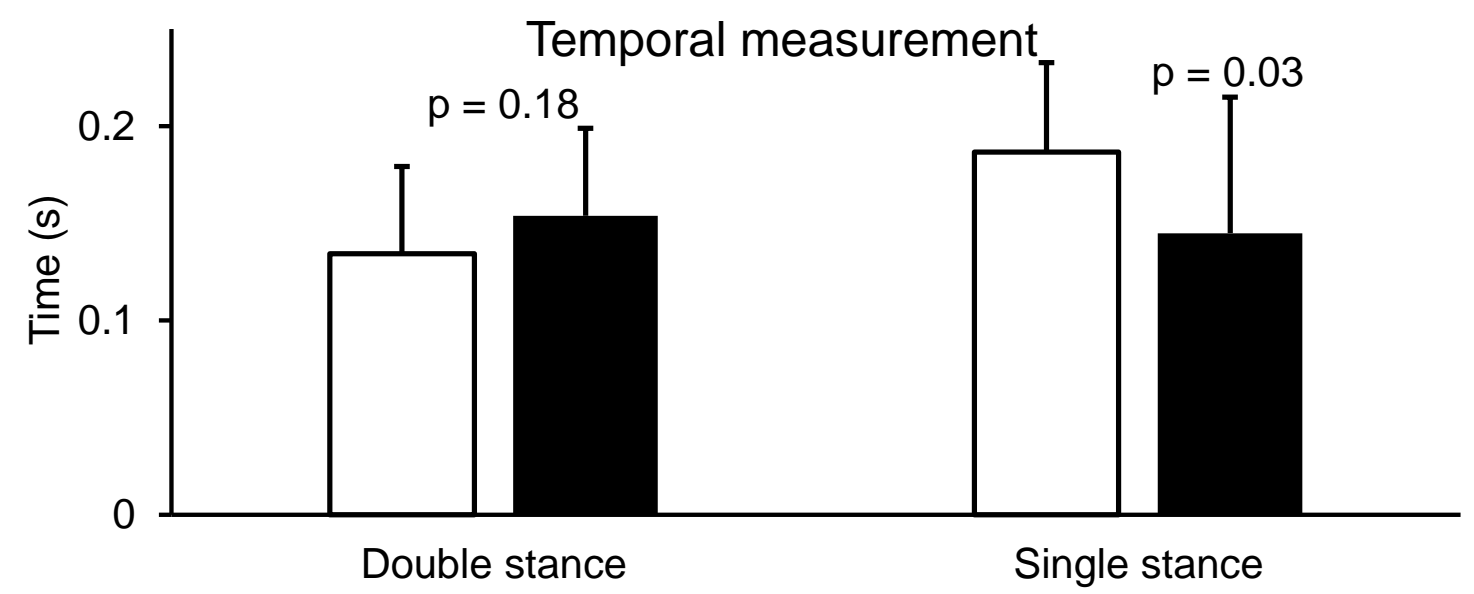

B.

Slip velocity

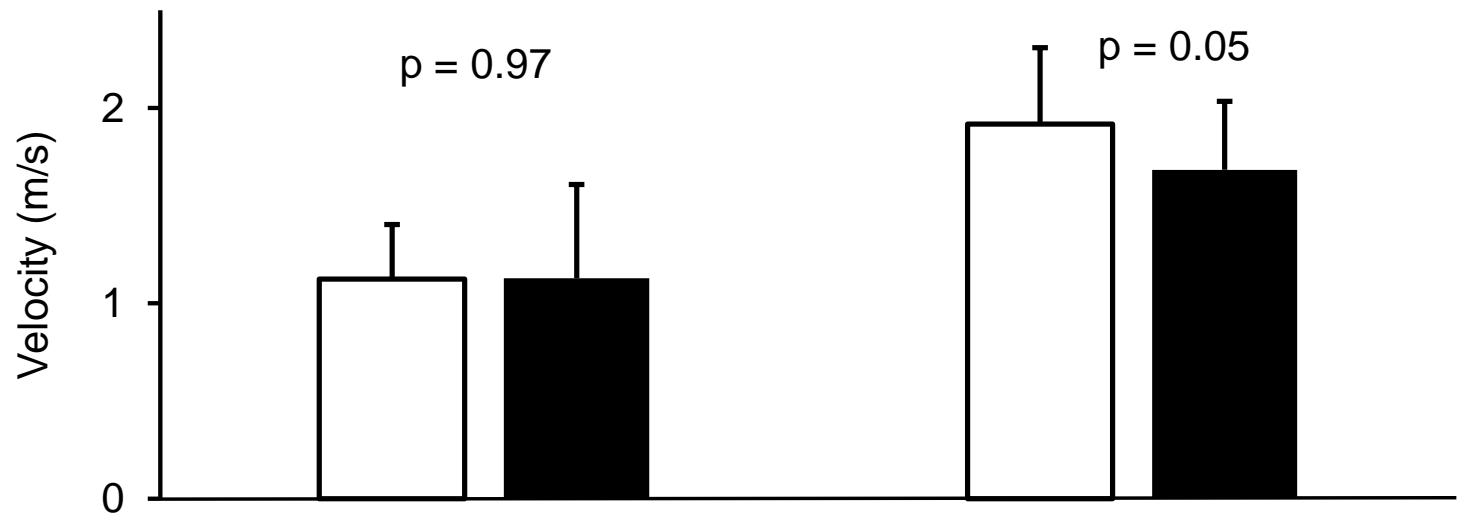

C.

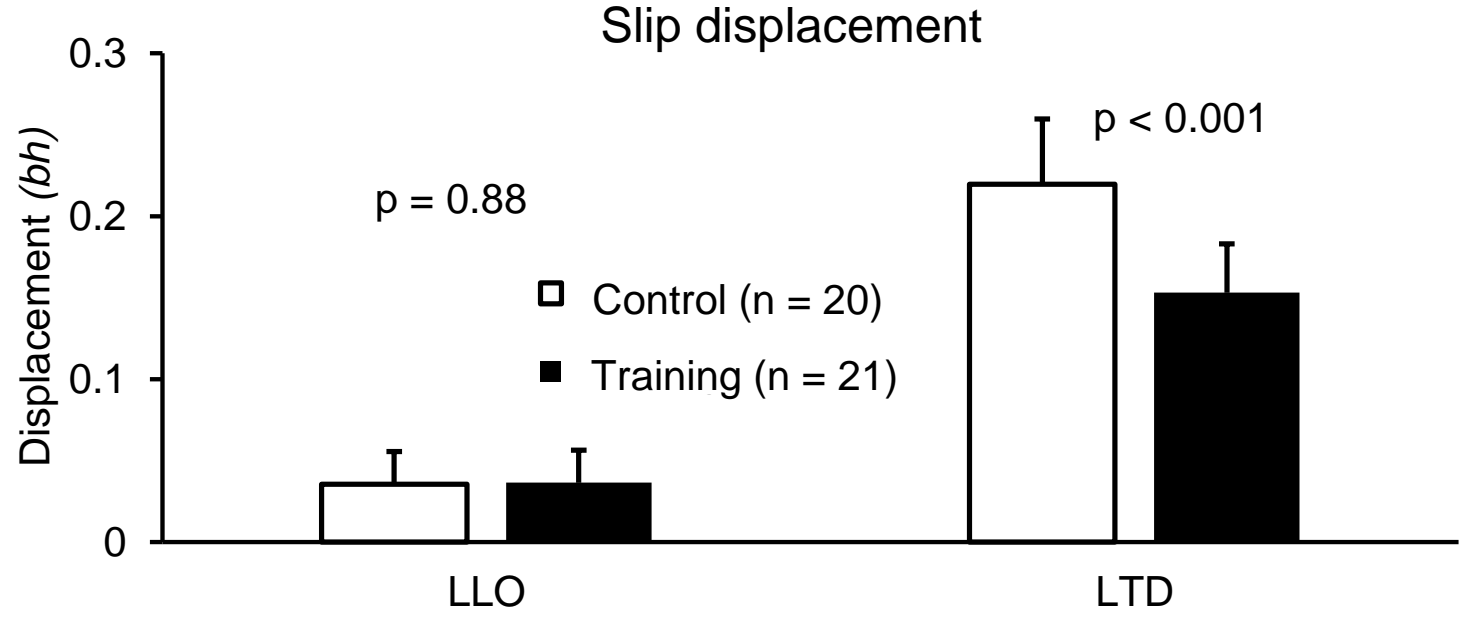

Figure 10

Comparisons of $(A)$ the duration of double stance (from right foot touchdown, or RTD, to left foot liftoff, LLO) and single stance phase (from left foot liftoff, LLO, to its touchdown, LTD), (B) the forward velocity and (C) anteroposterior displacement of the base of support (BOS) at LLO and LTD between the training and control groups during the overground slip. 


\section{DISCUSSION}

The purpose of this study was to investigate the overall effect and feasibility of a treadmill-based perturbation training paradigm consisting of 8 slip perturbations on reducing slip-related falls among young adults. The hypotheses of this study were: 1) Treadmill-based perturbation training would reduce the rate of falls and 2) Treadmillbased perturbation training would improve dynamic stability in response to an unannounced overground slip in healthy young adults. Our results revealed that the training group, who received the treadmill-based training, exhibited significantly lower fall rate and improved dynamic stability when exposed to the overground slip than the control group, who did not experience the perturbation training but a mocking training, lending support to both our hypotheses.

\subsection{Fall rate}

Our results supported the first hypothesis that a shortened, single-session treadmill-based perturbation training could reduce the fall rate in responding to a reallife like overground slip. Specifically, the control group had a fall rate of $65 \%$ which was significantly higher than that of the training group $(19.1 \%, p=0.003$, Fig. 8$)$. This finding is consistent with the ones from previous studies. For example, Yang et al. (2013) reported that the group who received a treadmill-based perturbation training protocol with up to 30 slips demonstrated significantly lower rate of falls ( $0 \%$ vs. $23.5 \%$ ) in comparison with those who did not experience the perturbation training.

The lower fall rate in the training group than in the control group implies that the fall resistance skills obtained from the treadmill training could be transferred to the overground slip. Previous studies suggested that humans can quickly adapt their gait 
pattern to potential slip surfaces when they are made aware of the possible slip perturbation during overground walking (Maki et al., 2008). Therefore, a key factor leading to the low overground-slip fall rate in the training group is the prior experience of slip perturbation during the perturbation training. Due to the prior experience, participants in the training group demonstrated a better and quicker reaction to the slip than the control group, as evidenced by the shorter duration of the single stance phase (Fig. 10A) and the better control of dynamic stability (Fig. 9) and BOS slip kinematics (Figs. 10B \& C) at LTD.

\subsection{Dynamic stability}

The results also verified the second hypothesis that a single-session treadmillbased perturbation training would significantly improve dynamic stability in response to a real-life like overground slip. At LTD during the overground slip, participants in the training group demonstrated significantly less instability in comparison with the control group although the stability values were negative for both groups at this time instant. Based on the FSR theoretical framework, a negative dynamic stability value implies that a person is in an instable state because the COM does not possess sufficient forward momentum to catch up to the BOS. Therefore, all participants in both groups experienced a backward balance loss as they landed their recovery step behind the leading foot (Fig. 9A). However, due to the significantly lesser instability in the training group than in the control group, participants in the training group fell much lesser than the control group (Fig. 8).

The improved stability due to treadmill-based training confirmed findings presented by previous studies. For instance, Yang et al., (2013) reported that young 
adults who underwent treadmill-based perturbation training were more stable than those who did not receive the training. Similarly, another study carried out by Liu et al., (2016) found the same results when comparing those who completed training to those who did not.

One of the most important functions of the central nervous system (CNS) is to maintain body balance and compensate for instabilities during locomotion. It is well known that with proper training, humans are adaptable to sudden or unexpected changes in environmental constraints during a range of activities from standing to locomotion (Rogers, Rogers, Takeshima, \& Islam, 2003). In the present study, participants in the training, but not the control group, improved their dynamic stability at LTD during the overground slip. In according to the FSR theory, dynamic gait stability can be altered through modifying the COM position and/or velocity relative to the BOS (Fig.1) (Pai \& Patton, 1997). In this study, the improvements in dynamic stability among the training group were achieved by adopting both strategies: placing their COM closer to the $\mathrm{BOS}$ and moving the COM faster comparing with the control group (Figs. 9A \& B).

However, dynamic stability along with COM position and velocity did not exhibit any significant group-related difference at RTD and LLO. This finding seems contradicted with the one from previous study in which young adults appeared to improve dynamic stability at RTD and LLO as well (Yang et al., 2013). One of the explanations to this discrepancy could be the intensity of the perturbation training. In detail, the number of perturbation slip on the treadmill was 8 in this study while participants in the previous study experienced up to 30 slips. Due to the difference in the training volume, the reduced version of the perturbation training adopted in this 
study may not be sufficient to induce the adaptive changes at RTD and LLO. Despite the less-intensive training in the present study, it still was able to improve dynamic stability to an extent at LTD which reduced the fall incidences in the training group, aligning with previous findings (Yang et al., 2013; Liu et al., 2016).

Another possible contributor to such a discrepancy could be related to the mechanisms of inducing the overground slip. In the previous study (Yang et al., 2013), the movable platform was about $2-3 \mathrm{~cm}$ higher than the surrounding walkway. Although it was not easily visible to participants due to its camouflage with the rest of the walkway, participants would have known the location of the slider after few trials of walking over it. Such information might result in some proactive responses to the impending slip, just as the changes in gait pattern at RTD (Yang et al., 2013). However, in the present study, the slider was on the exactly same elevation as the entire walkway in addition to the camouflage. Therefore, participants were completely blinded to the details of the slip including its location. The overground slip in the present was a real "novel" slip. Given the lack of the information about the location of the slippery surface, participants may not make any adaptive changes to their gait pattern until approximately $300 \mathrm{~ms}$ following the actual slip occurrence.

The third possible factor contributing to the difference in the stability at RTD and LLO between studies could be the experimental condition. Specifically, the lights were switched off during all overground trials in our study while the lights remained on during the previous study (Yang et al., 2013). The unaffected visual input in the previous study could have enabled the participants to visually see the location of the slider and further guess how the slider would work. In the present study, however, participants had nearly 
no visual input about the position of the movable platform and its working mechanisms due to the dimmed lights and the instruction that "keep looking forward during walking". This would prevent participants in this study from gaining any information or knowledge of the slider. As the result of the limited information of the impending slip, participants in the current study could not proactively but reactively respond to the impending slip.

\subsection{Temporal measurement and BOS kinematics}

Recovery stepping was executed much faster in the training group than in the control group (Fig. 10A, $p=0.029$ ). This was evident by the single stance temporal measurement results. Specifically, the single stance phase was much shorter in the training group in comparison with the control group $(0.145 \pm 0.068$ vs. $0.187 \pm 0.047$ sec, $p=0.029$, Fig. 10A). This result is in line with the one documented by Yang et al., (2013) and Liu et al., (2016), who found that those who received treadmill-based perturbation training would be able to take the recovery step much faster than those who do not experience the training when being exposed to the unexpected overground slip.

The importance of the quick execution of the recovery stepping could be explained by the necessary body reactions after a postural perturbation during gait. After a slip, to regain body balance and prevent an actual fall, one must generate quick

and sufficient corrective reactions during the recovery stepping (Cham \& Redfern, 2001; Yang et al., 2009). Two major reactions to a slip perturbation have been identified empirically (Cham \& Redfern, 2001) and analytically (Yang \& Pai, 2010). One reaction is a knee flexor moment which has the potential to pull the BOS towards the body's COM. Such reaction improves the dynamic stability and increases the chance of 
successfully recovering from the unexpected perturbation (Cham \& Redfern, 2001; Yang \& Pai, 2010).

Another reactive response is the extensor moment from the recovery leg providing sufficient anti-gravity support to prevent a limb collapse (Cham \& Redfern, 2001; Ding \& Yang, 2016). Adequate lower extremity muscle strength is demanded to execute both reaction strategies. If one can produce great muscle strength, the chance to retard and even reverse the falling after the slip would increase, reducing the probability of fall. However, participants in both groups are healthy young adults without any neurological or musculoskeletal diseases. It is reasonable to assume that the muscle strength may not be different between groups especially given the comparable demographic information between them (Table 1). Therefore, the speed at which one executes the recovery stepping becomes a key factor leading to falls after a slip. In the current study, participants in the training group were able to perform the recovery stepping significantly faster than among the control group. This would have played an important role to reduce fall incidents in this group.

Participants in the training group also exhibited a better control over the BOS kinematics during the overground slip than the control group. For instance, at LTD, the BOS travelled for a significantly shorter distance with a slower forward speed among the training group than the control group (Figs. 10B \& C). As dynamic stability was determined by the relative movement between the COM and BOS, the control of BOS movement during a slip is crucial to prevent a balance loss or fall (Pai \& Patton, 1997). In the present study, the improved BOS movements would bring the COM motion state closer to the limit against backward balance loss, thus enhancing dynamic stability in 
the training group. The improved stability in turn reduced the likelihood of falls in this group. Similarly, such a better control could be attributable to the prior slip experience on the treadmill trials among the training group. Our observations regarding the improved control of the BOS kinematics were in line with those from Yang et al. (2013), which also showed that the slip distances were significantly lower among individuals who received training when compared to those who did not.

\subsection{Implications}

Perturbation training is emerging as a viable modality to reduce laboratoryinduced falls (Maki et al., 2008; Bieryla \& Madigan, 2011; Yang et al., 2013). This approach is novel in that it concentrates on adaptation to externally induced perturbation instead of self-motivated improvements in physical conditioning, balance control, or volitional performance like other contemporary fall reduction methods (Mansfield et al., 2010; Yang et al., 2013; Liu et al., 2016). Perturbation training may possess some advantages against traditional exercise-based training. For example, it can induce gross errors that are involuntary in nature and therefore cannot be corrected by merely volitional performance. Experiencing such errors is essential for the CNS to recalibrate an existing internal representation of the environment (Maki et al., 2008) - in this case pertaining to the FSR - which provides the required basis for adaptive adjustments in the control of dynamic stability.

Our results, along with other previous studies (Yang et al., 2013; Liu et al., 2016), reinforce the notion that treadmill-based perturbation training is effective to reduce sliprelated falls. More importantly, our results revealed that a limited version of the perturbation training protocol can still improve the dynamic stability control and reduce 
the fall rate in response to an unannounced and novel overground slip. This also implies the efficient of our training paradigm. Therefore, a commercialized treadmillbased perturbation training protocol could be used to reduce the risk of falls among young and older adults. It has great potential to be clinically deployed to clinical settings, senior centers, or nursing homes. With the reduction of falls, the costs resulting from falls and relevant injuries will be lowered as well.

\subsection{Limitations}

This study represented some limitations. First, no baseline measurements of the body reactions to the overground slip were made. This might limit our ability to precisely target the effect (i.e., the changes in the outcome measurements from baseline to the post-training evaluation) resulting from the perturbation training. However, this study design was chosen in purpose. The key reason was the human's capability of learning. If participants were given a chance to experience the overground slip at the baseline test, they may have learned how to deal with the overground slip. This would influence or contaminate their reaction to the same overground slip during the post-training test. Therefore, the observed adaptive changes in the body reaction during the post-training test could be contributed by both the perturbation training and the learning effect from the baseline. It would be difficult, if not impossible, to isolate one from the other. On the other hand, the randomized controlled trail used in this study provided an ideal placebo group who did not experience any perturbation training but the mocking training on the same treadmill. Theoretically, any differences detected between groups should be solely attributable to the perturbation itself. The comparable demographic information between groups could further support this. 
Second, the muscle strength was not controlled in the current study. It was possible that the muscle strength could be mismatched between groups, thus resulting bias to our results. Whereas, the participants enrolled into this study were healthy young adults without any musculoskeletal diseases. It is reasonable to assume that the muscle strength could be comparable between groups. Another piece of indirect evidence supporting our assumption was the balanced demographic information between groups (Table 1).

Third, there was no recording of previous falls up to the training/testing date for the participants. The rate of previous falls sustained by participants could have been different between the two groups. Whereas, due to the nature of the randomized controlled design, it was reasonable to assume that the fall history could be similar between groups, limiting its effect on our findings. Further, the history of falls among healthy young adults may not closely relate to future falls, unlike among older adults (Heijnen \& Rietdyk, 2016). Hence, the lack of the collection of fall history in our participants may have little impact on our results.

Lastly, the retention effect of the training protocol in this study remains unknown. This is not a trivial issue since the longer the training effect can persist, the more impact the training paradigm could make. All these issues warrant further investigations based on a large sample size.

\subsection{Future directions}

Because the results of this study supported our hypotheses, there are many directions that future studies can take in this field of research. First, it was demonstrated that slip-related falls pose a danger to the elderly; therefore, a study of 
this nature could be applied to the elderly. Second, the training protocol explored in this study is only one of nearly countless options that may yield training effects to successfully mitigate fall risk. The present study merely represents an early attempt to simulate the spontaneous adaptive process observed in overground-slip training on a special treadmill. Therefore, to optimize the stimulus-response properties of this type of training could be another future direction. Third, once the effectiveness, efficacy, and dose-response features of perturbation training examined, it would also be beneficial to test this type of training with those suffering from a physical impairing disease such as Parkinson's or multiple sclerosis. No population is immune from experiencing a fall, so determining an efficient yet effective strategy to prevent falls in imperative to improve the quality of life for all individuals. 


\section{CONCLUSION}

In summary, our study indicated that a shortened treadmill-based perturbation training program could improve the dynamic stability control and decrease the rate of falls among young adults when they were exposed to a novel identical real-life like overground slip. This study used a total of eight treadmill-based slip perturbations, to our best knowledge, which represented the lowest perturbation training dose in this field of work. Such a conservative training dosage further proves that this type of training paradigm can be an effective and efficient one. While there are many other types of training that can reduce falls, this type of training may be one of the most applicable to the elderly who are at a high risk of sustaining a fall-related injury given its efficiency. Further research is needed for this type of training, but the given results derived from young adults in this study make a strong case that treadmill-based perturbation training can be applicable to other populations like individuals with movement disorders. 


\section{REFERENCES}

Alexander, B., Rivara, F., \& Wolf, M. (1992, July Vol.). The cost and frequency of hospitalization for fall-related injuries in older adults. Public Health Briefs, 82(7), 1020-1023.

Alvarez, K., Kirchner, S., Chu, S., Smith, S., Winnick-Baskin, W., \& Mielenz, T. (2015). Falls reduction and exercise training in an assisted living population. Journal of Aging Research , 1-4.

Benichou, O., \& Lord, S. (2016). Rationale for strengthening muscle to prevent falls and fractures: A review of the evidence. Calcif Tissue Int, 98, 531-545.

Bhatt, T., \& Pai, Y. (2008). Can observational training substitute motor training in preventing backward balance loss after an unexpected slip during walking? Journal of Neurophysiology, 99, 843-852.

Bhatt, T., Wening, J., \& Pai, Y. (2006). Adaptive control of gait stability in reducing sliprelated backward loss of balance. Experimental Brain Research, 170, 61-73.

Bieryla, K., \& Madigan, M. (2011). Proof of concept for perturbation-based balance training in older adults at a high risk for falls. Archives of Physical Medicine and Rehabilitation, 92, 841-843.

Borelli, G. (1680). De Motu Animalium. Rome: A. Bernabo.

Cham, R., \& Redfern, M. (2001). Lower extremity corrective reactions to slip events. Journal of Biomechanics, 34, 1439-1445.

Costs of falls among older adults. (2009, January 19). Retrieved from Center for Disease Control and Prevention: http://www.cdc.gov/HomeandRecreationalSafety/Falls/fallcost.html

de Leva, P. (1996). Adjusements to Zatsiorsky-Seluyanov's segment inertia paramters. Journal of Biomechanics, 29(9), 1223-1230.

Ding, L., \& Yang, F. (2016). Muscle weakness is related to slip-initiated falls among community-dwelling older adults . Journal of Biomechanics, 49, 238-243.

Englander, F., Hodson, T., \& Terregrossa, R. (1996). Economic dimensions of slip and fall injuries. Journal of Forensic Science, 41(5), 733-746.

Friedman, S., Munoz, B., West, S., Rubin, G., \& Fried, L. (2002). Falls and fear of falling: Which comes first? A longitudinal prediction model suggests strategies for primary and secondary prevention. Journal of the American Geriatrics Society, 50(8), 1329-1335. 
Granacher, U., Muehlbauer, T., Zahner, L., Golhofer, A., \& Kressig, R. (2011). Comparison of traditional and recent approaches in the promotion of balance and strength in older adults. Sports Medicine, 41(5), 377-400.

Han, L., \& Yang, F. (2015). Strength or power, which is more important to prevent sliprelated falls? Human Movement Science, 44, 192-200.

Hayes, W., Myers, E., Morris, J., Gerhart, T., Yett, H., \& Lipsitz, L. (1993). Impact near the hip dominates fracture risk in elderly nursing home residents who fall. Calcif Tissue INt, 52, 192-198.

Heijnen, M., \& Rietdyk, S. (2016). Falls in young adults: Perceived causes and environmental factors assessed with a daily online survey. Human Movement Science, 46, 86-95.

Hof, A., Gazendam, M., \& Sinke, W. (2005). The condition for dyanmic stability. Journal of Biomechanics, 38, 1-8.

Horak, F., \& Nashner, L. (1986). Central programming of postural movements: Adaptations to altered support-surface configurations. Journal of Neurophysiology, 55(6), 1369-1381.

Horlings, C., van Engelen, B., Allum, J., \& Bloem, B. (2008). A weak balance: the contribution of muscle weakness to postural instability and falls. Nature Reviews Neurology, 4, 504-515.

Kummel, J., Kramer, A., Giboin, L., \& Gruber, M. (2016). Specificity of balance training in healthy individuals: A systematic review and meta-analysis. Sports Medicine, $1-11$.

Kuo, A. (1995). An optimal control model for analyzing human postural balance. IEEE Transactions on Biomedical Engineering, 42(1), 87-101.

Liu, X., Bhatt, T., \& Pai, Y. (2016). Intensity and generalization of treadmill slip training: High or low, progressive increase or decrease? Journal of Biomechanics, 49, 135-140.

Luukinen, H., Herala, M., Koski, K., Honkanen, R., Laippala, P., \& Kivela, S. (2000). Fracture risk associated with a fall according to type of fall among the elderly. Osteoporos. Int., 11, 631-634.

Maki, B., Cheng, K., Mansfield, A., Scovil, C., Perry, S., Peters, A., . . Mcllroy, W. (2008). Preventing falls in older adults: New interventions to promote more effective change-in-support balance reactions. Journal of Electromyography and Kinesiology, 18, 243-254. 
Mansfield, A., Peters, A., Liu, B., \& Maki, B. (2010). Effect of a perturbation-based balance training program on compensatory stepping and grasping reactions in older adults: A randomized controlled trial. Physical Therapy, 90(4).

Melzer, I., Benjuya, N., \& Kaplanski, J. (2004). Postural stability in the elderly: A Comparison between fallers and non-fallers. Age and Aging, 33, 602-607.

Moreland, J., Richardson, J., Goldsmith, C., \& Clase, C. (2004). Muscle weakness and falls in older adults: A systematic review and meta-analysis. Journal of the American Geriatrics Society, 52, 1121-1129.

Pai, Y., \& Patton, J. (1997). Center of mass velocity - Position predictions for balance and control. Journal of Biomechanics, 30(4), 347-354.

Parkkari, J., Kannus, P., Palvanen, M., Natri, A., Vaino, J., Aho, H., . . Jarvinen, M. (1999). Majority of hip fractures occur as a result of a fall and impact on the greater trochanter of the femur: a prospective controlled hip fracture study with 206 consecutive patients. Calcif Tissue Int, 65, 183-187.

Patel, K., Phelan, E., Leveille, S., Lamb, S., Missikpode, C., Wallace, R., ... Turk, D. (2014). High prevalence of falls, fear of falling, and impaired balance in older adults with pain in the United States: Findings from the 2011 National Health and Aging Trends study. Journal of the American Geriatrics Society, 62(10), 18441852.

Patel, S., Tweed, K., \& Chinappen, U. (2005). Fall-related risk factors and osteoporosis in older women referred to an open access bone densitometry service. Age and Aging, 34, 67-71.

Pratt, J., \& Tedrake, R. (2006). Velocity based stability margins for fast bipedal walking . Fast Motions in Biomechanics and Robots, 1-27.

Prevention, C. f. (2015, August 15). Web-based injury statistics query and reporting systems (WISQARS). National Center for Injury Prevention and Control .

Rogers, M., Rogers, N., Takeshima, N., \& Islam, M. (2003). Methods to assess and improve the physical parameters associated with fall risk in older adults. Preventative Medicine, 36, 255-264.

Rubenstein, L. (2006). Falls in older people: epidemiology, risk factors and strategies for prevention. Age and Aging, 35(2), ii37-ii41.

Sessoms, P., Wyatt, M., Grabiner, M., Collins, J.-D., Kingsbury, T., Thesing, N., \& Kaufman, K. (2014). Method for evoking a trip-like response using a treadbillbased perturbation during locomotion. Journal of Biomechanics, 47, 277-280.

Shimada, H., Obuchi, S., Furuna, T., \& Suzuki, T. (2004). New intervention program for preventing falls among frail elderly people: The effects of perturbed walking 
exercise using a bilateral separated treadmill. American Journal of Physical Medicine and Rehabilitation, 83, 493-499.

Stevens, J., Corso, P., Finkelstein, E., \& Miller, T. (2006). The costs of fatal and nonfatal falls among older adults. Injury Prevention, 12, 290-295.

Townsend, M. (1985). Biped gait stabilization via foot placement. Journal of Biomechanics, 18, 21-38.

Vellas, B., Wayne, S., Romero, L., Baumgartner, R., \& Garry, P. (1997). Fear of falling and resitriction of mobility in elderly fallers. Age and Ageing, 26, 189-193.

Winter, D., Palta, A., \& Frank, J. (1990). Assessment of balance control in humans. Medical Progress Through Technology, 16((1-2)), 31-51.

Yang, F., \& Pai, Y. (2010). Role of individual lower limb joints in reactive stability control following a novel slip in gait. Journal of Biomechanics, 43(3), 397-404.

Yang, F., Anderson, F., \& Pai, Y. (2007). Predicted threshold against backward balance loss in gait. Journal of Biomechanics, 40(4), 804-811.

Yang, F., Bhatt, T., \& Pai, Y. (2009). Role of stability and limb support in recovery against a fall following a novel slip induced in different daily activities. Journal of Biomechanics, 42, 1903-1908.

Yang, F., Bhatt, T., \& Pai, Y. (2013). Gernalization of treadmill-slip training to prevent a fall following a sudden (novel) slip in over-ground walking. Journal of Biomechanics, 46(1), 63-69.

Yang, F., King, G., Dillon, L., \& Su, X. (2015). Controlled whole-body vibration training reduces risk of falls among community-dwelling older adults. Journal of Biomechanics, 48, 3206-3212.

Yang, F., Passarillo, F., \& Pai, Y. (2008). Determination of instantaneous stability against backward balance loss: Two computational approaches. Journal of Biomechanics, 41, 1818-1822. 


\section{VITA}

Patrick Anthony Cereceres is the son of a Mexican immigrant and of a secondgeneration Latina. He was born and raised in the small town of Canutillo, Texas, just outside of El Paso on the state line with New Mexico. After Patrick graduated from high school, he attended the University of Texas at Austin (UT Austin), where he received a Bachelor of Education in Kinesiology. While at UT Austin, Patrick served as the President of the Alpha Pi Chapter of Phi Kappa Theta Fraternity.

After graduation from UT Austin, Patrick went home to Canutillo and was accepted to the Master's of Kinesiology program in the College of Health Science at the University of Texas at El Paso. Throughout the entirety of his post-graduate career, Patrick has worked as a teaching assistant for the Department of Kinesiology, where he helped teach Exercise Physiology, Anatomical Kinesiology, and Fitness Programs \& Appraisal. He has been working closely with Dr. Yang in the Stanley E. Fulton Biomechanics and Motor Behavior lab for the past year and a half. After graduation, he plans to pursue a PhD in Exercise Physiology.

Contact information:

Address: PO Box 1581

Email: pacereceres@miners.utep.edu

Cellphone: (915) $490-1181$

This thesis was typed by Patrick Anthony Cereceres. 